\title{
Emergence of planar cell polarity from the interplay of asymmetric interaction and tissue-level gradients
}

\author{
Divyoj Singh ${ }^{1,2}$, Mohit Kumar Jolly ${ }^{1, *}$ Mohd. Suhail Rizvi ${ }^{3, *}$ \\ 1 Centre for BioSystems Science and Engineering, Indian Institute of \\ Science, Bangalore 560012, India \\ 2 Undergraduate Programme, Indian Institute of Science, Bangalore \\ 560012 , India \\ 3 Department of Biomedical Engineering, Indian Institute of Technology, \\ Hyderabad 502284, India
}

*Corresponding authors: suhailr@bme.iith.ac.in and mkjolly@iisc.ac.in

\begin{abstract}
Planar cell polarity (PCP) - asymmetric localization of proteins at cell-cell interface - is essential for embryonic development and physiological functions. Abnormalities in PCP can lead to neural tube closure defects, misalignment in hair follicles etc. Thus, decoding the mechanism responsible for PCP establishment and maintenance remains an open fundamental question. While various molecules - broadly classified into "global" and "local" modules - have been well investigated, their necessity and sufficiency in explaining PCP and connecting their perturbations and defects in experimentally observed patterns has not been examined. Here, we develop a minimal model that captures the proposed features of these two modules- a global tissue level gradient and local asymmetric distribution of protein complexes. Our model results suggest that while polarity can emerge in absence of a gradient, the gradient can provide the direction of polarity as well as offer robustness for maintenance of PCP in presence of stochastic perturbations. We also recapitulated swirling patterns (seen experimentally) and the features of non-domineering autonomy, using only three free parameters in the model - protein binding rate, concentration of proteins forming heterodimer across cell boundaries and steepness of gradient. Our results explain how self-stabilizing asymmetric localisations in presence of tissue-level gradient can lead to robust PCP patterns in diverse biological systems and reveals the minimal design principles for a polarized system.
\end{abstract}

\section{Introduction}

Epithelial tissues form the outer most layer of most organs including skin. They not only function as a protective layer for the inner tissues but also take part in several physiological functions including absorption, secretion, excretion and filtration [1. Epithelial cells usually manifest two major types of polarity: Apico-basal polarity and Planar cell polarity (PCP). Apical-basal polarity refers to how certain cellular components and functions are asymmetrically distributed between two opposite surfaces of an epithelial sheet. PCP, on the other hand, refers to organization in a direction parallel to the plane of the epithelial sheet 2 . 
PCP is an evolutionarily conserved phenomenon which is required for early developmental events such as neural tube closure and later in the formation of functional organs [3]. It has been a topic of extensive scientific investigation, especially in the model organism Drosophila melanogaster. In Drosophila, PCP has been investigated to understand its molecular mechanisms and the fallouts of its disruptions in the physiology of several organs, such as hair bristles on wing, abdomen and ommatidia in eye [4] .

Disruption in PCP can lead to several developmental abnormalities such as neural tube defects, idiopathic scoliosis, skeletal dysplasia manifested as as short limbs and craniofacial anomalies 2 and tracheal ciliary malfunction [5]. PCP is essential also for auditory and vestibular function of the vertebrate inner ear. It determines how mechanosensory hair cells sense sound or motion [6]. Thus, defects in PCP have been associated with hearing loss 7 . Therefore understanding the multi-scale dynamics of PCP establishment and maintenance is a fundamental question.

The molecular players involved in the establishment of PCP in various organisms have been well studied. Even though the interactions among the proteins involved in PCP are quite intricate and not necessarily well demarcated, they have been categorized into two groups, also known as modules: Global and Core [2].

The global module contains two atypical cadherins,d Fat (Ft) and Dachsous (Ds), the kinase Four-jointed $(\mathrm{Fj})$, and the myosin Dachs(D) 8. Ft and Ds from adjacent cells can form heterodimers at cell-cell interface. Their affinity for each other can be modulated by Fj 9 11. Dachs co-localises with Ds and generates active forces at the apical end, thus regulating organ shape [12]. Proteins of global modules are usually present in tissue-level expression gradients 13 15]. These tissue level gradients can provide the global cue to $\mathrm{PCP}$ machinery which helps alignment of the PCP with the axis of the organ [4].

On the other hand, the core/local module consists of Frizzled (Fz), Flamingo (Fmi), Van Gogh (Vang), Prickle-spiny-legs (Pk), Dishevelled (Dsh) and Diego (Dgo) 16 which interact with one another, some intercellularly and some intracellularly, by forming complexes. Similar to Ft-Ds heterodimer across cell-cell boundary, Fmi from two neighbouring cells form complexes. The trans-membrane protein $\mathrm{Fz}$ recruits the cytosolic proteins Dsh and Dgo, and localises in proximity of Fmi in one of the two cells. Another trans-membrane protein Vang recruits cytosolic protein Pk close to Fmi on the other cell. These interactions among the core module have been argued to be sufficient for establishing a local order (polarity in two adjacent cells) 2, 17]. 
A
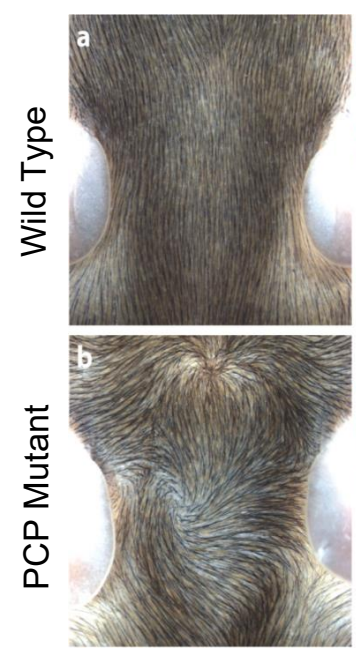

C
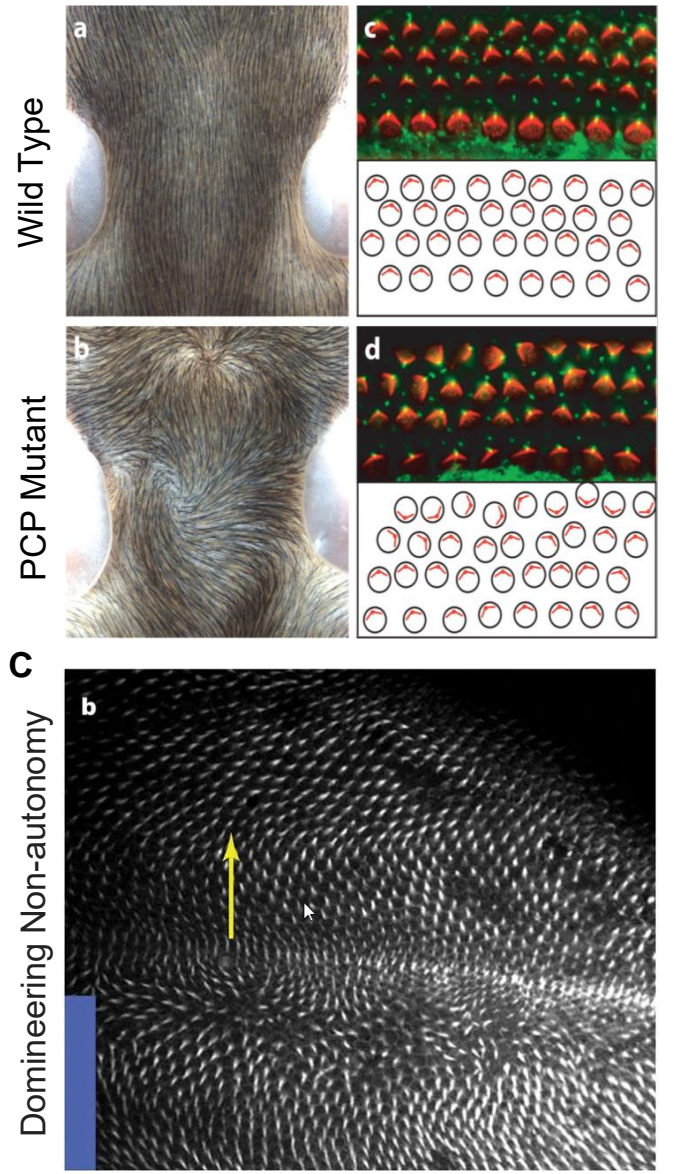

\section{B}
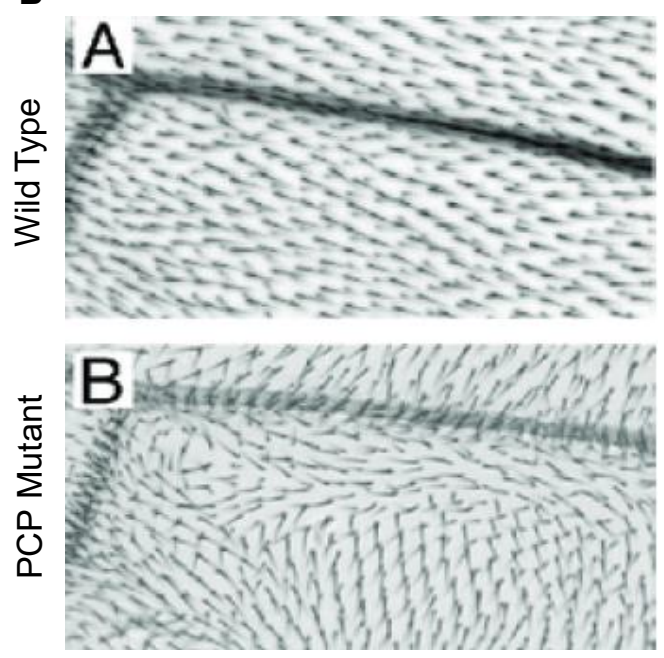

D

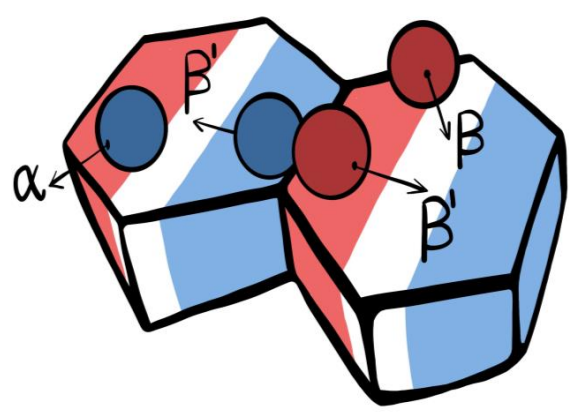

Figure 1. Introduction to Planar cell polarity and the model. A) Examples of PCP in mammals (mouse skin and inner ear) taken from 18. B Example of PCP in wing of Drosophila melanogaster taken from 19 C) Experimental image showing non-autonomous domineering taken from 20. D) Schematic to depict the protein binding and unbinding kinetics and heterodimer formation.

An understanding of PCP crucially requires establishing the relative contributions of global agencies such as gradients and local interactions like heterodimer formation in the emergence and maintenance of polarity 21,22. Most models have thought of global interactions as a means for providing directional cues 23. Local interactions like formation of heterodimer have been considered as output of formation of polarity and used as a readout of polarization 24. In principle the global interactions such as tissue-level gradient can be responsible for formation of polarity and its maintenance in the presence of perturbation. The local interactions can also lead to the asymmetric distribution of proteins across the cell and thus lead to formation of polarity. How redundant or synergistic the roles of these two features - asymmetric localisation and tissue-level gradient - are remains to be decoded.

Given the complex interactions between molecules within and across modules, mathematical modelling has been an alternative essential tool to account for experimental abnormalities and make further testable experimental predictions. Despite the two-dimensional nature of epithelial tissue, most mathematical models of the PCP have been formulated for one dimension (for example 25 26]), with exceptions where the biological question studied required a two-dimensional framework 24,27,29]. There are 
broadly two classes of models for PCP. One class takes into account the mechanistic details of the system and explains examples of PCP establishment and its disruption in specific organs (see 30 for review). While they provide useful inputs for the experimental contexts for which they are formulated, their generalizability across parameter sets or biological contexts is limited. The other class are phenomenological models that apply to a wider variety of cases and parameter ranges because they ignore some mechanistic details but account for the pattern formation 28. They don't focus on several intriguing experimental observations like disruption in polarity when a gene is deleted from a region. These models provide limited insights that can be directly tested experimentally.

Here, we integrate the strengths of both these kinds of models. We incorporate minimal mechanistic relations with the goal of capturing general dynamical properties of the system, together with focus on specific interesting experimental observations. Towards this, we focus on the inter-cellular interactions of membrane associated proteins to form dimer complexes (homo/hetero) which is a common feature in core and global module despite the involvement of a diversity of proteins. Our formalism is developed in 1D and 2D; thus overcoming limitations of several previous models, which fail to capture the quintessential features of PCP dynamics in 1D framework [25, 26]. Using the minimal model with only three parameters we demonstrate that PCP can emerge even without any external gradient. We find multiple roles for the protein expression gradients in addition to being the global cue in PCP. We also explain patterns/defects noted on deleting specific proteins from a region in a tissue as well as swirling patterns and non-homogeneous polarization in tissue.

\section{Materials and Methods}

\section{Basic assumptions}

We assume epithelial tissue to be a non motile confluent layer of cells. For simplicity, we ignore the three dimensional organ specific epithelial architecture and assume identical apical-basal geometry of the cells. Planar cell polarity being a two dimensional phenomenon, this assumption of identical apical and basal cross-sections of the cells is a fair simplification and has remained a common feature across all the PCP modeling approaches. In order to keep the analysis limited to PCP we also do not take into account any cellular rearrangements, cell movements or remodeling in the our model. However, it can be seen that these biophysical aspects can also be easily integrated in the model, though some care is warranted vis-a-vis the timescales involved in these biophysical processes, the biochemical protein interactions and protein turnover kinetics.

\section{Protein binding and transport kinetics}

In order to build the model we focus on the two members of the global module of PCP, Fat (or Ft) and Dachsous (or Ds), atypical cadherins which interact across the membranes of two adjacent cells (inter-cellular interaction). The modeling framework, however, is equally applicable to the core module as well (see Discussion). Both of Ft and Ds, if present in cytoplasm, can bind to the cell membrane and if bound to cell membrane can detach from there to go into cytoplasm. We consider that in an 'isolated' cell (a hypothetical situation where inter-cellular interactions are ignored) the attachment and detachment of these cadherins follow the law of mass action and the kinetic rates of attachment and detachment are $\alpha$ and $\beta$, respectively. It needs to be pointed out that here, for the sake of simplicity, we are taking these rate constants to be same for both proteins but the argument can easily be generalized to disparate rate 
coefficients.

Next, we assume that the time scales of protein translation and degradation are much larger than the timescales of protein kinetics of binding and unbinding of proteins to the cell-membrane. Therefore we ignore the changes in levels of total protein concentrations in a cell due to translation and degradation. We assume diffusion of protein inside a cell is happening at faster timescale compared to protein heterodimer formation, therefore, the concentrations of both proteins is taken to be uniform in a cell.

Finally, we take inter-cellular protein interaction at the cell membrane into account in the following manner. The cadherin nature of these proteins is well understood and they have been shown to form Ft-Ds heterodimer across cell membranes. Therefore, when a Ft (or Ds) is bound to the cell membrane its rate of detachment goes down due to its interaction with the Ds (or Ft) from neighboring cell. We take this into account by modifying the rate of detachment as

$$
\beta^{\prime}=\beta(1-\gamma c)
$$

where $c$ is the concentration of Ds (or Ft) at the membrane of the neighboring cell. $\gamma$ here is acts as the facilitation parameter for repressing the unbinding of Ft from the cell membrane by Ds of neighboring cell (or vice-versa) and forming a heterodimer.

With this simple biochemical kinetic assumption we now move on to the formulation of the dynamics of PCP in the tissue. First, we will describe it for a one dimensional tissue.

\section{One dimensional model}

In the 1-D model, we assume a single layer of cells. The total concentrations of Ft and Ds in cell is kept constant thanks to the assumption mentioned previously.

Since in 1D each cell has only two ends, the concentrations of membrane bound Ft at either sides of the cells are denoted by $F t_{L}(i)$ (amount of $F t$ on left edge of the cell at location $i$ ), $F t_{R}(i)$ (amount of $F t$ on right edge of the cell at location $i$ ). Similarly, for Ds these quantities are denoted by $D s_{L}(i)$ and $D s_{R}(i)$.

The equations describing the concentrations of membrane bound proteins are

$$
\begin{aligned}
& \dot{F} t_{L}(i)=\underbrace{\alpha\left(F t_{T}(i)-F t_{0}(i)\right)}_{\text {Binding to Cell membrane }}-\underbrace{\beta F t_{L}(i)\left(1-\gamma D s_{R}(i-1)\right)}_{\text {Unbinding from Cell membrane }}, \\
& \dot{F} t_{R}(i)=\alpha\left(F t_{T}(i)-F t_{0}(i)\right)-\beta F t_{R}(i)\left(1-\gamma D s_{L}(i+1)\right), \\
& \dot{D} s_{L}(i)=\alpha\left(D s_{T}(i)-D s_{0}(i)\right)-\beta D s_{L}(i)\left(1-\gamma F t_{R}(i-1)\right), \\
& \dot{D} s_{R}(i)=\alpha\left(D s_{T}(i)-D s_{0}(i)\right)-\beta D s_{R}(i)\left(1-\gamma F t_{L}(i+1)\right),
\end{aligned}
$$

where $F t_{0}(i)=F t_{L}(i)+F t_{R}(i)$ and $D s_{0}(i)=D s_{L}(i)+D s_{R}(i)$ are the total membrane bound protein in the $i^{\text {th }}$ cell. The two terms on the right hand sides of the equations represent the rates of protein binding and unbinding (which is dependent on the protein concentrations of other protein in the neighboring cell), respectively. Here, $F_{T}(i)$ and $D s_{T}(i)$ stand for the amount of total (cytoplasmic+membrane bound) Ft and Ds present in the cell and can be dependent on the cell position in the tissue, that is $i$ or $x$ in the discrete and continuum representations, respectively.

In this work, we consider the cell polarity to be dependent on the asymmetric localization of the two proteins in the cell. We write the asymmetry or polarity of the two proteins in the $i^{\text {th }}$ cell as the difference between the protein concentrations on right and left edges of the cell, that is

$$
\begin{array}{r}
p_{\mathrm{Ft}}(i)=F t_{R}(i)-F t_{L}(i) \\
p_{\mathrm{Ds}}(i)=D s_{R}(i)-D s_{L}(i)
\end{array}
$$


which represents the asymmetry in the protein localization on cells edges.

Finally, using these definitions of protein polarity we define

$$
\Delta p=p_{\mathrm{Ft}}-p_{\mathrm{Ds}}
$$

as the cell polarity. This is the crucial observable in the system as it leads to overall polarity in the system. Please note that there is no reason for defining $\Delta p$ as the cell polarity and not $-\Delta p$. The results obtained in this paper are not dependent on this choice. $-\Delta p$ just reflects polarization in opposite direction.

To simulate the 1D model, we considered an array of 500 cells and numerically solved the system of ordinary differential equations using solve_ivp function from scipy package(version 1.6.2) in Python with adaptive time stepping.

\section{Effect of noise in the system}

\section{Noise in protein kinetics}

To study the effect of noise in the system due to protein kinetics, we write stochastic equations for the system. For the stochastic simulations for the 1D case, we modify the equations by adding a noise term in the form of Langevin equation as:

$$
\begin{aligned}
& \dot{F} t_{L}(i)=\alpha\left(F t_{T}(i)-F t_{0}(i)\right)-\beta F t_{L}(i)\left(1-\gamma D s_{R}(i-1)\right)+\eta_{i}^{L}(F t, t) \\
& \dot{F} t_{R}(i)=\alpha\left(F t_{T}(i)-F t_{0}(i)\right)-\beta F t_{R}(i)\left(1-\gamma D s_{L}(i+1)\right)+\eta_{i}^{R}(F t, t) \\
& \dot{D} s_{L}(i)=\alpha\left(D s_{T}(i)-D s_{0}(i)\right)-\beta D s_{L}(i)\left(1-\gamma F t_{R}(i-1)\right)+\eta_{i}^{L}(D s, t) \\
& \dot{D} s_{R}(i)=\alpha\left(D s_{T}(i)-D s_{0}(i)\right)-\beta D s_{R}(i)\left(1-\gamma F t_{L}(i+1)\right)+\eta_{i}^{R}(D s, t)
\end{aligned}
$$

where the noise terms ( $\operatorname{such}$ as $\eta_{i}^{L}(F t, t)$ ) are uncorrelated along time $t$, position of the cell in the tissue $i$, position of the edges in a cell $L$ or $R$, and also in terms of the protein concentrations $F t$ and $D s$, that is

$$
\eta_{i}^{P}\left(A, t_{m}\right) \eta_{j}^{Q}\left(B, t_{n}\right)=\delta_{i, j} \delta_{t_{m}, t_{n}} \delta_{P, Q} \delta_{A, B}
$$

where $P$ and $Q$ can take values $L$ and $R, A$ and $B$ stand for the proteins Ft and Ds, and $\delta$ is the Kronecker delta function. In order to implement this we select a random number for each cell edge $T\left(i, P, A, t_{m}\right) \in \mathcal{N}\left(0, \sigma^{2}\right)$ from a standard Normal distribution with mean $\mu=0$ and standard deviation 1 . Using this, we write the noise term in the equation as

$$
\eta_{i}^{Q}(A, t)=\tilde{\eta}_{0} \times T\left(i, P, A, t_{m}\right)
$$

where $\tilde{\eta}_{0}$ represents the amplitude of the noise or the magnitude of stochasticity in the system. Because this is not a deterministic system, we performed several instances of simulations and calculate the average polarization. Therefore we took average over 50 simulations.

\section{Noise in total protein concentration of Ft and Ds}

To study the effect of noise due to cell division, death, protein production and degradation, we added noise in the total protein concentration of both the proteins in following way:

$$
\begin{aligned}
F t_{T}(i) & =\rho(1+\sigma(F t, i)) \\
D s_{T}(i) & =\rho(1+\sigma(D s, i))
\end{aligned}
$$


where the noise terms (such as $\sigma(F t, i)$ ) are uncorrelated along position of the cell in the tissue $i$, in terms of the protein concentrations $F t$ and $D s$, that is

$$
\sigma(A, i) \sigma(B, j)=\delta_{i, j} \delta_{A, B}
$$

where $A$ and $B$ stand for the proteins Ft and Ds and $i$ and $j$ stand for the index (position) of the cell. To implement noise in total protein concentration, we selected a random number $T(A, i) \in \mathcal{N}(0,1)$ from a Standard Normal distribution with mean 0 and standard deviation 1 . Using this, we write the noise term in the equation as

$$
\sigma(A, i)=\tilde{\sigma}_{0} \times T(A, i)
$$

where $\tilde{\sigma}_{0}$ represents the amplitude of the noise or the magnitude of stochasticity in the system. Because this is not a deterministic system, we performed several instances of simulations and calculate the average polarization. Therefore we took average over 50 simulations.

\section{Two dimensional model}

For the 2D model, we used a lattice of immotile cells formed of regular hexagons with each cell having six neighbors as opposed to two in 1D. Following same approach as in $1 \mathrm{D}$, we write equations for dynamics of protein concentration for a cell at $\mathbf{x}$ :

$$
\begin{aligned}
& \dot{F t}\left(\mathbf{x}, \theta_{i}\right)=\underbrace{\alpha\left(F t_{T}(\mathbf{x})-F t_{0}\right)}_{\text {Binding to Membrane }}-\underbrace{\beta F t\left(\mathbf{x}, \theta_{i}\right)\left(1-\gamma D s\left(\mathbf{x}+\Delta \mathbf{x}_{\theta_{i}}, \theta_{i}+\pi\right)\right)}_{\text {Unbinding from Membrane }} \\
& \dot{D} s\left(\mathbf{x}, \theta_{i}\right)=\alpha\left(D s_{T}(\mathbf{x})-D s_{0}\right)-\beta D s\left(\mathbf{x}, \theta_{i}\right)\left(1-\gamma F t\left(\mathbf{x}+\Delta \mathbf{x}_{\theta_{i}}, \theta_{i}+\pi\right)\right)
\end{aligned}
$$

where

$$
\begin{aligned}
& F t_{0}(\mathbf{x}, t)=\sum_{\theta_{i}=0}^{2 \pi} F t\left(\mathbf{x}, \theta_{i}, t\right) \\
& D s_{0}(\mathbf{x}, t)=\sum_{\theta_{i}=0}^{2 \pi} F t\left(\mathbf{x}, \theta_{i}, t\right)
\end{aligned}
$$

are the total membrane bound protein concentration in the cell. Here we sum over the protein concentration at the 6 angles $\theta_{i}=0=2 \pi, \pi / 6, \pi / 3, \pi / 2,2 \pi / 3$ and $5 \pi / 6$. ( 6 parts of discretised cell membrane). $F t_{T}(\mathbf{x}, t)$ and $D s_{T}(\mathbf{x}, t)$ are the total (cytoplasmic + membrane bound) protein levels in the cell located at $\mathbf{x}$, and $F t\left(\mathbf{x}+\Delta \mathbf{x}_{\theta_{i}}, \theta_{i}+\pi, t\right)$ represents the protein $F t$ in the neighboring cell.

Similar to the one-dimensional model, we can define the planar cell polarity (PCP) of a protein in a cell in terms of the asymmetric distribution of that protein on the cell membrane. This definition of PCP for protein Ft translates as a vector with following two components of $\mathbf{P}_{F t}$ :

$$
\begin{aligned}
& P_{F t}^{x}(\mathbf{x}, t)=\sum_{\theta_{i}=0}^{2 \pi} F t\left(\mathbf{x}, \theta_{i}, t\right) \cos \theta_{i} \\
& P_{F t}^{y}(\mathbf{x}, t)=\sum_{\theta_{i}=0}^{2 \pi} F t\left(\mathbf{x}, \theta_{i}, t\right) \sin \theta_{i} .
\end{aligned}
$$

Finally we define

$$
\Delta \mathbf{P}=\mathbf{P}_{F t}-\mathbf{P}_{D s} .
$$

To simulate the $2 \mathrm{D}$ system, we take a $2 \mathrm{D}$ hexagonal lattice of $50 \times 50$ cells and solve the equations for the Ft and Ds dynamics at cell boundaries using Euler's method. 


\section{Parameter values}

To understand the system analytically and reduce the modelling parameters, we non-dimensionalize the system using the following characteristic quantities

1. $\tau=1 / \beta$ for time,

2. $1 / \gamma$ for protein levels.

This results in just three free parameters in the system- Protein binding rate $(\alpha)$, total protein concentrations $F t_{T}$ and $D s_{T}$. These parameters along with the initial conditions can control the wide variety of patterns seen in PCP. We have kept the value of $\alpha$ fixed at 10 in the results shown unless specified otherwise. The protein concentrations are used as a control parameter to study the nature of polarization and the effects of protein gradient expressions, protein loss and gain.

\section{Results}

\section{Polarity can emerge even in the absence of gradient in protein expression}

In PCP, tissue-level gradients of Ft and Ds are well documented 20,31 33, however, their exact role and the mechanism of their action is not completely understood. Thus, we first ask whether polarity can emerge in absence of any tissue-level gradient. In absence of any tissue-level gradient; $F t_{T}$ and $D s_{T}$ are independent of $i$. Here for simplicity we have kept their levels equal at $\rho$, referred to as total protein concentrations (membrane+cytoplasm). The results remain valid even if these levels are unequal.

We first observe that the total concentrations of membrane bound Ft and Ds increase in an equal amount with $\rho$, that is $F t_{L}+F t_{R}=D s_{L}+D s_{R}=\rho_{0}$. However, there is a threshold of total protein concentration, $\rho_{c}$, after which an increase in $\rho$ results in abrupt increase in the membrane bound protein levels $(\mathbf{F i g} 2 \mathrm{C})$. Below the threshold protein concentration, both the proteins localise on both sides of the cell in equal amount. Therefore there is no polarization of the cells (Fig $2 \mathrm{D}$ and Fig S1). But once we cross the threshold concentration, that is $\rho>\rho_{c}$, Ft gets bound to one side of the cell and Ds on the other side, resulting in non-zero polarization $(\mathbf{F i g} 2 \mathrm{D}$ and Fig S1). The direction of polarization here is determined by the initial conditions. The presence of threshold is due to non-linearity arising from the inter-cellular interactions and heterodimer formation between the proteins across the membranes of two neighboring cells $($ Fig 2 E).

Next we studied the dependence of the membrane bound protein concentrations $\left(\rho_{0}\right)$ as a function of the protein binding rate $(\alpha)$. Since the threshold protein concentration can depend on the binding rate $\alpha$ we generated a phase diagram of polarization as a function of effective protein binding rate, $\alpha$ and total protein concentration $(\rho)$. The phase diagram shows the existence of a polarized state $(\Delta p \neq 0)$ and an unpolarised state $(\Delta p=0)$. The boundary separating the two states shows that with an increasing $\alpha$ the threshold concentration of proteins required for the polarization decreases.

In order to get an intuitive understanding of the establishment of polarization above the critical protein concentrations, we looked at the continuum limit of our discrete model of 1D epithelium (see Appendix for details) 34]. In the continuum limit the size of the single cell is taken to be much smaller as compared to the length scale of the tissue. In this limit we get partial differential equations in place of ordinary differential equations (2)-(5) we have described earlier In the case of uniform protein expression levels, we get the following equation for the polarization (in non-dimensional form)

$$
\Delta \dot{p}=-\left(1-\rho_{0}\right) \Delta p
$$


where $\Delta p=$ is the cell polarity as described in equation $(8)$ and $\rho_{0}$ is the total concentration of the membrane bound proteins. This equation tell us that if $\rho_{0}>1$ the cell polarity is going to attain a non-zero value. The equation above may indicate that the cell polarity $(\Delta p)$ will increase indefinitely. This, however, is not the case since as soon as at $\rho_{0}=\Delta p / 2$ (which is also seen in Fig. 2 $2 \mathrm{E}-\mathrm{F}$ ) the two proteins get localized on two edges of the cell and further change in concentrations stops. Thus, we show that the inter-cellular interactions, in the form of heterodimer formation between Ft and Ds, are sufficient for the emergence of polarity in the absence of any tissue level expression gradient.

\section{Stochasticity results in partial loss of tissue level coordination in PCP}

We have seen that in a completely deterministic system (with no source of stochasticity or noise) the tissue shows polarization. Noise, however, is usually a ubiquitous feature in biological systems 35 . In the context of PCP, the noise can arise from different sources and can have different timescales as well. One source of noise are the cellular processes like protein production and degradation; cell division, growth, birth and death which can affect the total protein concentration of Ft and Ds in each cell. Hence, there will be variation in these protein concentrations across the tissue. Therefore, we looked at the impact of such stochastic effects on the polarization in the tissue.

We have incorporated noise in the equations as described earlier in (16). For simplicity, we have considered the noise amplitude to be not very large as compared to $\rho$, the average concentration of protein in each cell. We start with calculating average polarization in the tissue $(\Delta p)$ (averaged over multiple simulations) in the system for a range of noise amplitude $\left(\tilde{\sigma}_{0}\right)(\mathbf{F i g} 3 \mathrm{~A})$. We observe that the tissue starts losing the polarization if the level of noise is beyond a threshold level of noise.

To understand the properties of the dynamics of this system, we performed stability analysis in the continuum model [34]. The stability analysis tells us that the both the unpolarized state (below the threshold total protein concentration) and the polarized state (above the total protein concentration) are stable against homogeneous perturbations. However the polarized state is unstable against non-homogeneous perturbation.

The other source of noise in PCP arises from the nature of the protein binding and unbinding from the membrane or the inter-cellular interactions at the cell-cell interface. To study the effect of this source of noise, we incorporated noise in the equations as described earlier in methods (10)- $(12)$ ). Again, we have considered the noise amplitude to be not very large as compared to $\beta / \gamma$, the characteristic rate of the protein kinetics in this system. We again calculated average polarization in the tissue $(\Delta p)$ (averaged over multiple simulations) in the system for a range of noise amplitude $\left(\tilde{\eta}_{0}\right)$ (Fig $\left.\mathrm{S} 2 \mathrm{~A}\right)$. We see similar behaviour as seen for the noise in total protein concentration. Once the noise amplitude $\left(\tilde{\eta}_{0}\right)$ is higher than a threshold level, the tissue starts loosing polarization.

The question which still remains unanswered is whether the presence of noise results in reduction in polarization of all the cells or parts of the tissue polarized in different directions. To address this, we calculated the polarity of each cell in the tissue for different amplitudes of noise $\left(\tilde{\sigma}_{0}\right)$ and $\left(\tilde{\eta}_{0}\right)$. Note, that these results are for one instance of simulation.

When the noise amplitude is small $\left(\tilde{\sigma}_{0}=10^{-5}\right)$, tissue remains polarized uniformly. The direction of polarity of the tissue is determined by the initial conditions (Fig $3 \mathrm{~B}$ ). As we increase the noise amplitude $\left(\tilde{\sigma}_{0}=10^{-4}\right)$, some of the cells are polarised in opposite direction $(\mathbf{F i g} 3 \mathrm{C})$. On further increasing the amplitude of noise $\left(\tilde{\sigma}_{0}=10^{-3}\right)$, the overall polarity reduces due to patches of tissue polarising in opposite directions 
A

C
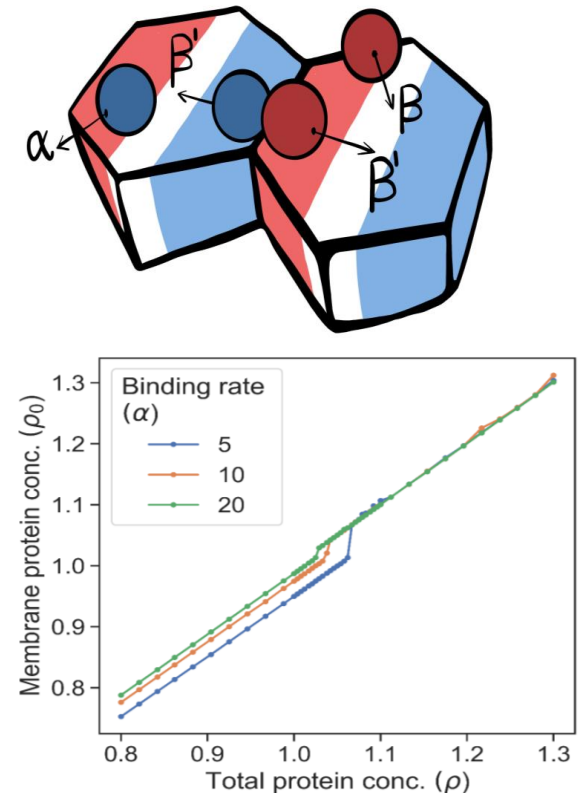

E

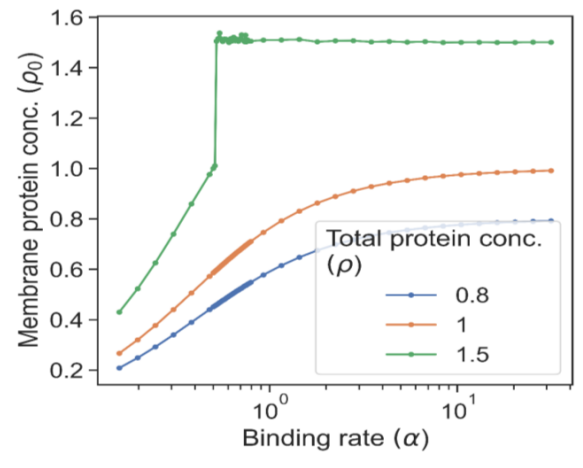

B

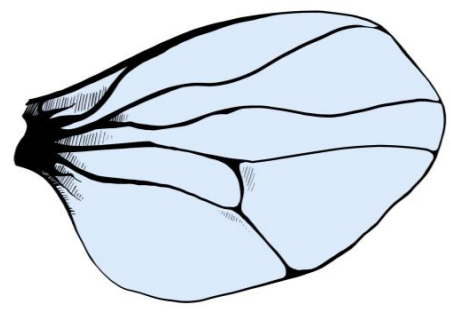

D

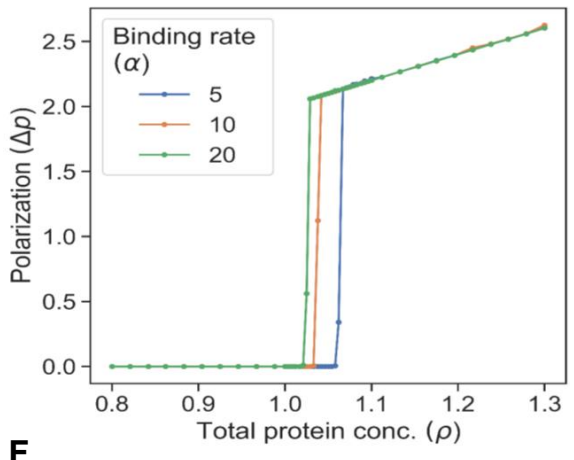

$\mathbf{F}$

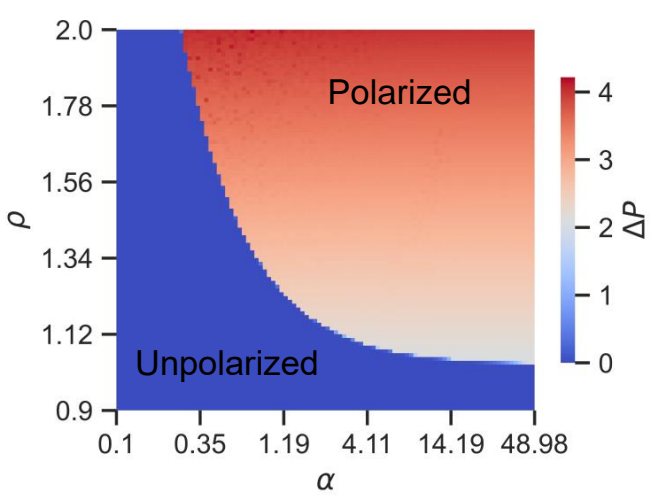

Figure 2. Emergence of polarity due to inter-cellular interactions in absence of any tissue level gradient in expression. A) Schematic describing the binding and unbinding of the proteins at the membrane and its regulation by heterodimer formation. $\alpha$ is the binding rate of the protein towards the cell membrane and $\beta$ is the unbinding rate. $\beta$, is the reduced unbinding rate if the heterodimer is formed. $\mathbf{B}$ ) Schematic to show uniform expression levels of both proteins across the tissue. Note that the results in this figure are from the one-dimensional model. C) Membrane protein concentration (of Ft and Ds) $\left(\rho_{0}\right)$ at the steady state against total protein concentration $(\rho)$. D) Plot of polarization magnitude $(\Delta p)$ against total protein concentration $(\rho)$. Tissue gets polarized above a threshold of total membrane concentration. E) Plot of Membrane protein concentration (of Ft and Ds) $\left(\rho_{0}\right)$ at steady state as a function of protein binding rate $(\alpha)$ at different total protein concentrations $(\rho)$. F) Phase plot of Polarization $(\Delta p)$ as a function of binding rate $(\alpha)$ and total protein concentrations $(\rho)$ shows the existence of a polarized (red) and unpolarised state (blue) 
(Fig 3 D). The tissue as a whole eventually loses polarity if the noise amplitude is too high (Fig $3 \mathrm{E})$ because of all cells polarizing in opposite directions randomly. This, however, needs to be pointed out that in the case with high amplitude of noise even though the average polarization in the whole tissue vanishes the individual cells remain polarized. Similar results are seen when noise is added to the protein kinetics equations (Fig $[$ S2 B-E) These observations show that the noise only influences the overall coordination of the the polarity in the tissue with minimal effect on PCP of individual cells.

\section{Swirling PCP patterns are possible in the absence of gradient in expression}

Swirling patterns in the PCP have been shown in several experimental works 20, especially when the global cue, the tissue level expression gradients, are not present 12 . Therefore, we also attempted to see if non-uniform polarization of the tissue is possible to achieve with this model. Since such pattern is in itself a two-dimensional phenomenon, we resolved to study the system in 2D hexagonal lattice. Two dimensional hexagonal lattice is relatively closer to actual epithelial geometry as compared to its 1D counterpart. We start with the case where the total protein concentration of both the proteins is uniform throughout the tissue and is equal to $\rho(\mathbf{F i g} 6$ A). Similar to the 1D case, we observe that the tissue gets polarized if the total protein concentration is above the threshold value (Fig $4 \mathrm{~B}$ ). Although, this critical protein concentration in 2D is not necessarily equal to that in $1 \mathrm{D}$ (see 34 for more detailed analysis). When we study the effect of noise (both due to variation in total protein concentration and protein kinetics) in our two dimensional model we get non-homogeneous polarization in the tissue. We observe different kinds of patterns some of which have been famously observed in PCP [12]. First we observe swirling patterns and singularities of polarization in which polarization in multiple cells either originate or terminate or rotate around a point (Fig 4 C). The amplitude of noise in total protein concentration is very small for this case $\left(\tilde{\sigma}_{0}=10^{-5}\right)$. This is an interesting finding and explains the swirling patterns observed in experimental systems by Axelrod et al. (2020) [36] as shown in (Fig 6 D).

Next, we observe a case where polarization is in different directions in patches (Fig 6 E). The amplitude of noise in total protein concentration is higher as compared to earlier $\left(\tilde{\sigma}_{0}=10^{-1}\right)$. This captures the experimental findings observed by Matakatsu et al. (2004) 37. This shows that in 2D the PCP direction is not always along any particular directions, such as the tissue axis. The continuum model also predicts the existence of a critical point and non-uniform polarization in $2 \mathrm{D}$ system.

\section{Gradients in protein concentrations facilitate PCP and provide global cue}

As mentioned earlier, the proteins of global module of PCP are expressed in tissue level 12. These gradients have been studied extensively in the context of the wing development in Drosophila (Fig 5A). Therefore, we also looked at the effect of the expression gradients of the proteins on PCP establishment and its alignment with tissue axis. For simplicity, in our model, we start with studying the effects of a linear gradient of Ft. Gradient of Ds also have similar effects and results remain unaltered. The choice for studying the linear gradient stems from inspiration from experimental studies which demonstrated the presence of gradients which are either linear or can be represented by piece-wise linear approximations. Several other modelling works 25, 26 have also taken gradients to be linear in the position of cell in the tissue. 

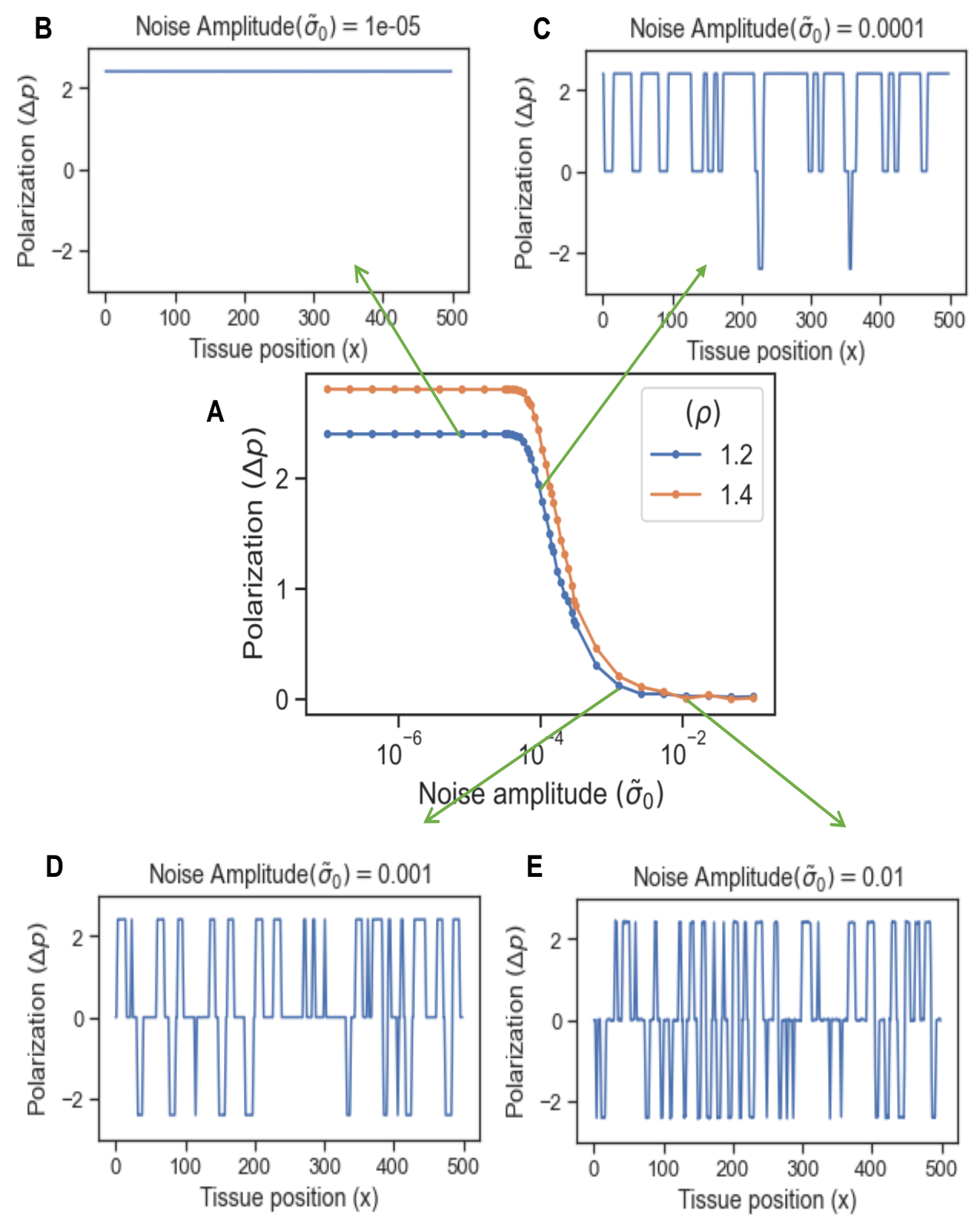

Figure 3. Effect of noise in total protein concentrations on polarization A) Polarization $(\Delta p)$ as function of noise amplitude $\left(\tilde{\sigma}_{0}\right)$ for different total protein concentrations $(\rho)$. B) Polarization $(\Delta p)$ in the tissue when the level of noise is negligible $\left(\tilde{\sigma}_{0}=10^{-5}\right)$. C) Polarization at high amplitude of noise $\left(\tilde{\sigma}_{0}=10^{-4}\right)$, some parts of the tissue are aligned in the opposite direction D) At higher noise amplitude $\left(\tilde{\sigma}_{0}=10^{-3}\right)$, the average polarity of the tissue reduces $\mathbf{E})$ At very high noise amplitude $\left(\tilde{\sigma}_{0}=10^{-2}\right)$, the tissue loses overall polarization as different parts of the tissue are aligned in opposite direction. 
bioRxiv preprint doi: https://doi.org/10.1101/2021.11.30.468750; this version posted December 1.2021. The copyright holder for this preprint (which was not certified by peer review) is the author/funder, who has granted bioRxiv a license to display the preprint in perpetuity. It is made available under aCC-BY-NC-ND 4.0 International license.
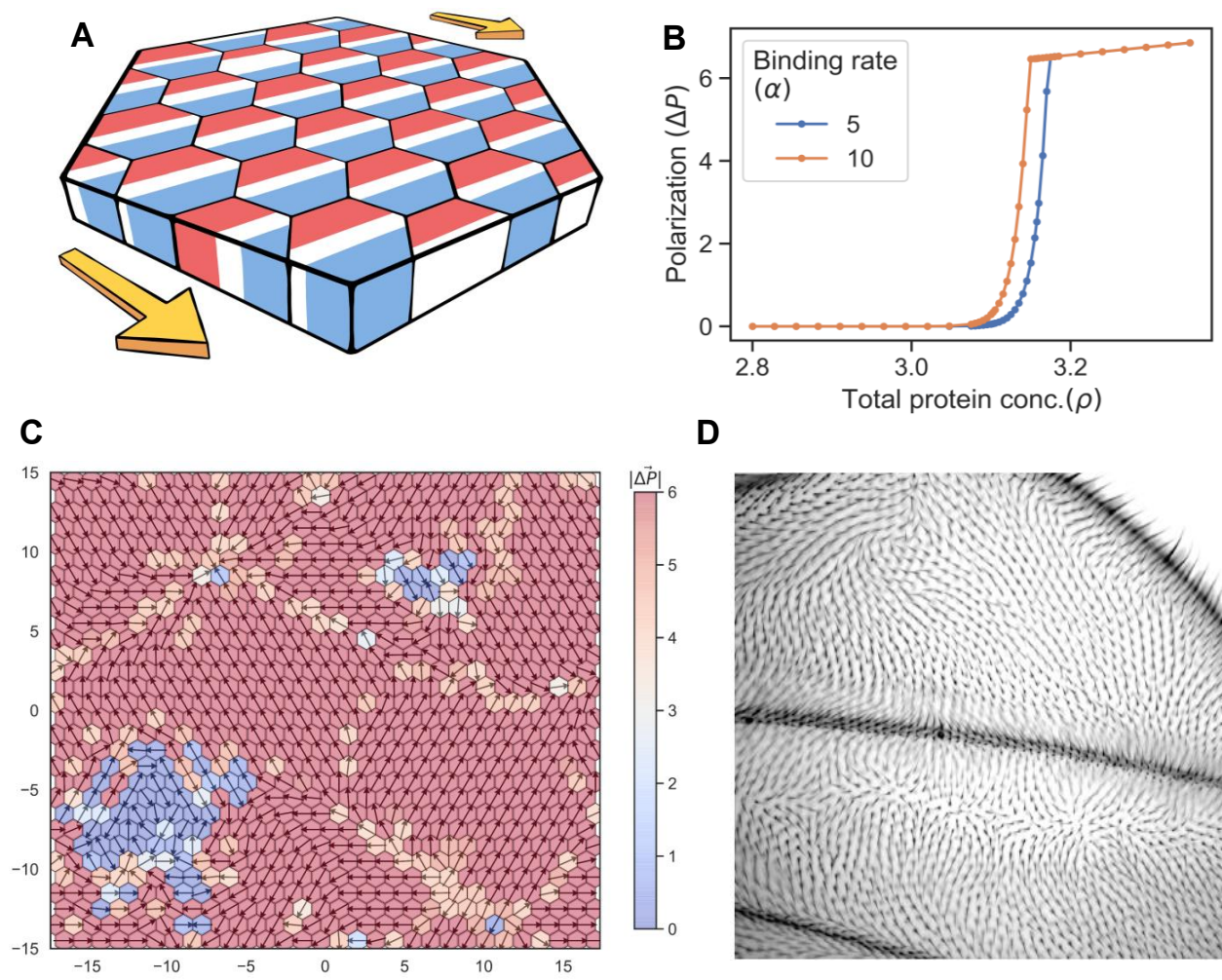

D

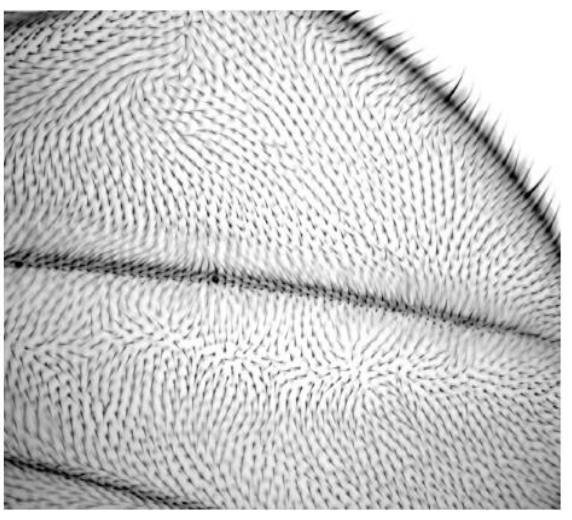

\section{$E$}

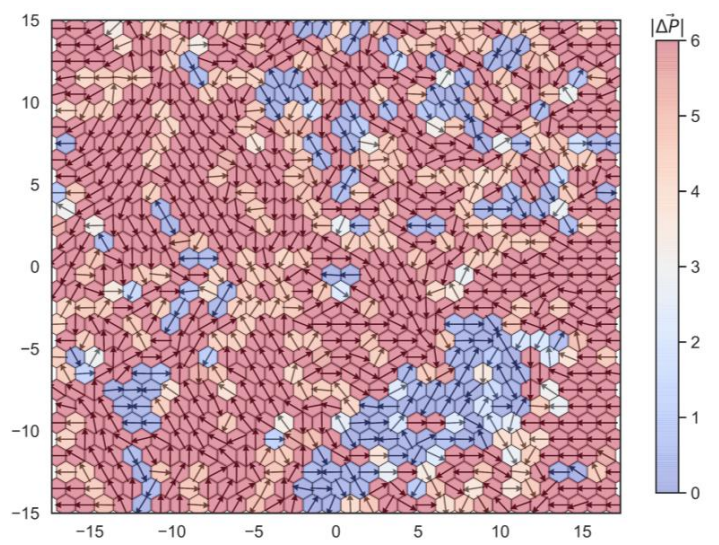

$\mathbf{F}$

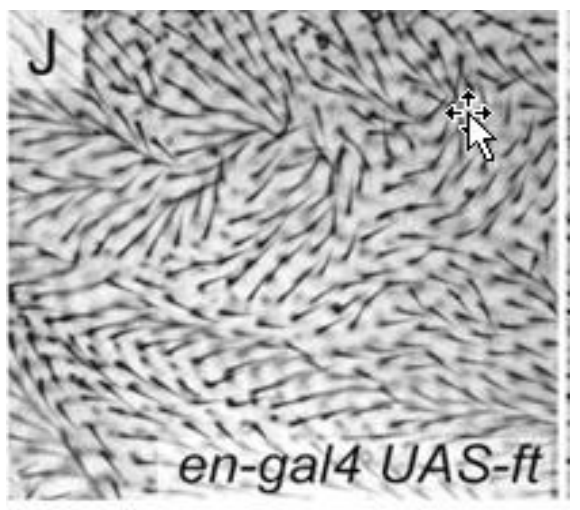

Figure 4. Polarization in 2D lattice for uniform protein expression of Ft and Ds: A) Schematic to describe the model of uniform protein expression of Ft and Ds in the 2D layer of tissue. B) Polarization $(\Delta P)$ as a function of total protein concentration $(\rho)$. The system starts getting polarized above a critical value of total protein concentration. C) Swirling patterns are observed when noise is introduced in the total protein concentration $\left(\tilde{\sigma}_{0}=10^{-8}\right)$ D) Experimental image of global mutant taken from Axelrod et al. 2020 [36] which shows and swirling patterns E) Patches of tissue polarized in different direction when the amplitude of noise in total protein concentration is higher $\left(\tilde{\sigma}_{0}=10^{-3}\right)$. F) Experimental image taken from 37 depicting a case where we observe patches of tissue polarized in different directions. 
We incorporate the linear gradient in Ft expression such that the total protein concentration of $\mathrm{Ft}$ varies in the tissue as

$$
F t_{T}=\rho+\epsilon x(i)
$$

with $\epsilon \ll \rho$ and $\rho$ is the concentration of $\mathrm{Ft}$ at some reference location in the tissue. Concentration of Ds is taken to be uniform throughout the tissue and is equal to $\rho$. Here, $\epsilon$ decides the steepness or the strength of gradient in the tissue and $x(i)$ is the position of the cell(having index $i$ ) in the tissue. We studied the effect of expression gradients in both $1 \mathrm{D}$ and $2 \mathrm{D}$. In both cases the results are largely similar. If $\epsilon$ is positive then cell towards right has more total concentration of $\mathrm{Ft}$ and the one on the left has lesser concentration as shown in Fig 5 A and Fig S4 A. Here we present the results from one dimensional model, whereas the results from 2 dimensional model are presented in the supplementary information.

Recall that in the absence of any gradient, the tissue remains unpolarized if the total protein concentration of Ft and Ds are below the critical concentrations. This, however, is not the case with gradient. In the presence of gradient, polarization can emerge below the critical point as well (Fig 5 B and Fig S4 C). Although for protein levels below critical concentration the cell polarization is weak. The magnitude of polarity in Ft and Ds depends on $\epsilon$ the steepness of the expression gradients and also on $\rho$. Interestingly, polarization of $\mathrm{Ft}\left(p_{\mathrm{Ft}}\right)$ is in the opposite direction as that of the gradient. We can understand this observation in the following way. If a cell on the right has more Ft concentration than the one on left, then Ds will localise more towards right because of the inter-cellular heterodimer formation. Since this process will happen in all cells, Ft from the cell towards right of each cell will start localising towards left side of the cell to form heterodimer with Ds of adjacent cell. This feedback loop amplifies their initial differences over time and will result in eventual localization of $\mathrm{Ft}$ towards left side of all the cells and Ds on the right side.

If the total protein concentration of $\mathrm{Ft}$ and Ds is above the threshold protein concentration, the tissue polarizes due to inter-cellular interactions but the gradient decides the direction of polarization. Due to the combined effect of gradient and spontaneous polarization, in this scenario, the cells get strongly polarized (compare the values on y-axes of Figs. $5 \mathrm{~B}$ and $5 \mathrm{C}$ ). Polarization of $\mathrm{Ft}$ is again opposite to the gradient direction. Even slight gradient is able to decide the direction of polarization and the tissue then polarizes due to the feedback loop of inter-cellular interactions (Fig $5 \mathrm{C}$ and Fig S4 D). We studied the effect of noise in protein kinetics to understand how noise disrupts the directional cue provided by gradient $(\mathbf{F i g} \mathrm{S} 3 \mathrm{~A})$. We calculated the polarity of $\mathrm{Ft}\left(p_{\mathrm{Ft}}\right)$ and Ds $\left(p_{\mathrm{Ds}}\right)$ as function of gradient of $\mathrm{Ft}(\epsilon)$ for different noise amplitudes $\left(\tilde{\eta}_{0}\right)$. We observe that the gradient still decides the direction polarity in presence of noise. Though because of noise, some cells polarize in opposite direction and this result in reduction of overall polarity.

Next, we study how gradient affects the robustness of polarization in presence of noise. We calculated the polarity of $\mathrm{Ft}\left(p_{\mathrm{Ft}}\right)$ and Ds $\left(p_{\mathrm{Ds}}\right)$ as function of noise amplitudes $\left(\tilde{\eta}_{0}\right)$ for different gradient steepness of Ft $(\epsilon)(\mathbf{F i g}$ S3 B $)$. We observe that higher the gradient, higher noise amplitude is needed system to loose the polarization. Therefore, gradient helps in maintenance of the polarized state and system with higher gradient is more robust to perturbations. Finally, we study the effect of total protein concentration $(\rho)$ on polarization of $\mathrm{Ft}\left(p_{\mathrm{Ft}}\right)$ and Ds $\left(p_{\mathrm{Ds}}\right)$ for various gradient amplitudes $(\epsilon)(\mathbf{F i g} 5 \mathrm{D}$ and $\mathbf{F i g} \mathrm{S} 4 \mathrm{E})$. An increase in the gradient shifts the transition between weak and strong polarization of the cells to smaller values of $\rho$.

To understand how gradient facilitates the polarization analytically, we use the continuum model [34]. We solve the system for the case where total protein 
concentration is below the critical protein concentration. From the calculations we get following for the steady state:

$$
\Delta p=\frac{\rho_{0}}{\left(2 \rho_{0}-1\right)} \frac{2 \alpha \epsilon}{2(\alpha+1)} .
$$

This equation tells us that the tissue will always be polarized in presence of the gradient. The slope of polarization $(\Delta p)$ vs $(\epsilon)$ is determined by $\left(\rho_{0}\right)$ which in turn is determined by the protein binding rate $(\alpha)$ and total protein concentration of Ds $(\rho)$. We also performed the stability analysis which tells us that the polarization is stable against non-homogeneous perturbations in the presence of a gradient.

These results show that expression gradients of the proteins in the PCP also play other roles in addition to providing global cue to align PCP direction with tissue axis. Gradient, not only helps in polarity establishment when overall protein levels are low but also stabilizes the polarized state as well.

\section{Down-regulation of $\mathrm{Ft}$ from a region in absence of gradient leads to exponentially decaying isotropic polarity defects.}

In experimental investigations to identify the role of the protein interactions and their non-cell autonomous effects the protein expression levels are perturbed from a select regions of the tissue 20]. In one such experimental strategy Ft or Ds are downregulated or upregulated from a region of the tissue and its fall out is observed in the cells of that region as well as outside or it. In order to recapitulate this scenario in silico, we set the total concentration of $\mathrm{Ft}$ in a region of the 1D tissue (the left half) to be zero as shown in Fig 6A while keeping it to be uniform and nonzero in the right half of the tissue. One the other hand the the total protein concentration of Ds in the tissue is kept uniform and non-zero. To deal with the discontinuity at the deletion boundary we modify the equations by adding an extra term in them as described here:

$$
\dot{F} t_{L}(i)=\underbrace{\alpha\left(F t_{T}(i)-F t_{0}(i)\right)}_{\text {Binding to Cell membrane }}-\underbrace{\beta F t_{L}(i)\left(1-\gamma D s_{R}(i-1)\right)}_{\text {Unbinding from Cell membrane }}-\underbrace{\zeta\left(F t_{L}(i)-F t_{R}(i)\right)^{3}}_{\text {to prevent overshooting }}
$$

Here, the third term $\zeta\left(F t_{L}(i)-F t_{R}(i)\right)^{3}$ makes sure that the concentrations do not overshoot to unrealistic values and prevents numerical instability near the deletion boundary. This term is set to zero otherwise because its not required otherwise and doesn't alter the results. At the deletion boundary, this term prevents the overshooting of protein concentration on one side of the cell with respect to the other.

When the total protein concentration is above the critical concentration,we observe that deleting $\mathrm{Ft}$ from a region induces a polarization which decays as we move away from the deletion boundary (Fig 6 B). Ft from the cell towards right of the deletion boundary binds to the Ds from the cell towards left of the deletion boundary. This leads to more Ft aligning towards left and Ds towards right.As we move away from the deletion boundary, the effect of this inter-cellular interactions decays. We fitted the decay of polarity to a decaying exponential function and calculated the decay length of the polarization, i.e., the length or number of cells after which the polarization reduces to $e^{-1}$ of its value at the deletion boundary. Next, we calculate this decay length as a function of total protein concentration $(\mathbf{F i g} 6$ C). Finally, we plot the polarization as a function of total protein concentration at several distances from the deletion boundary. Specifically we calculate the polarization as function of total protein concentration at the deletion boundary and at points which are 5,10,50 cells away from the deletion boundary and for the case when there is no deletion in the tissue (all cells have equal Ft 
A

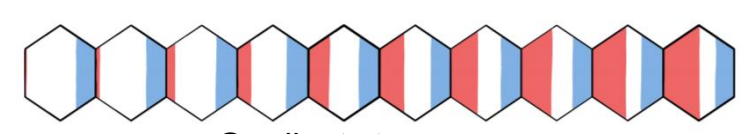

$\varepsilon \rightarrow$ Gradient steepness

B

Effect of gradient below the critical point
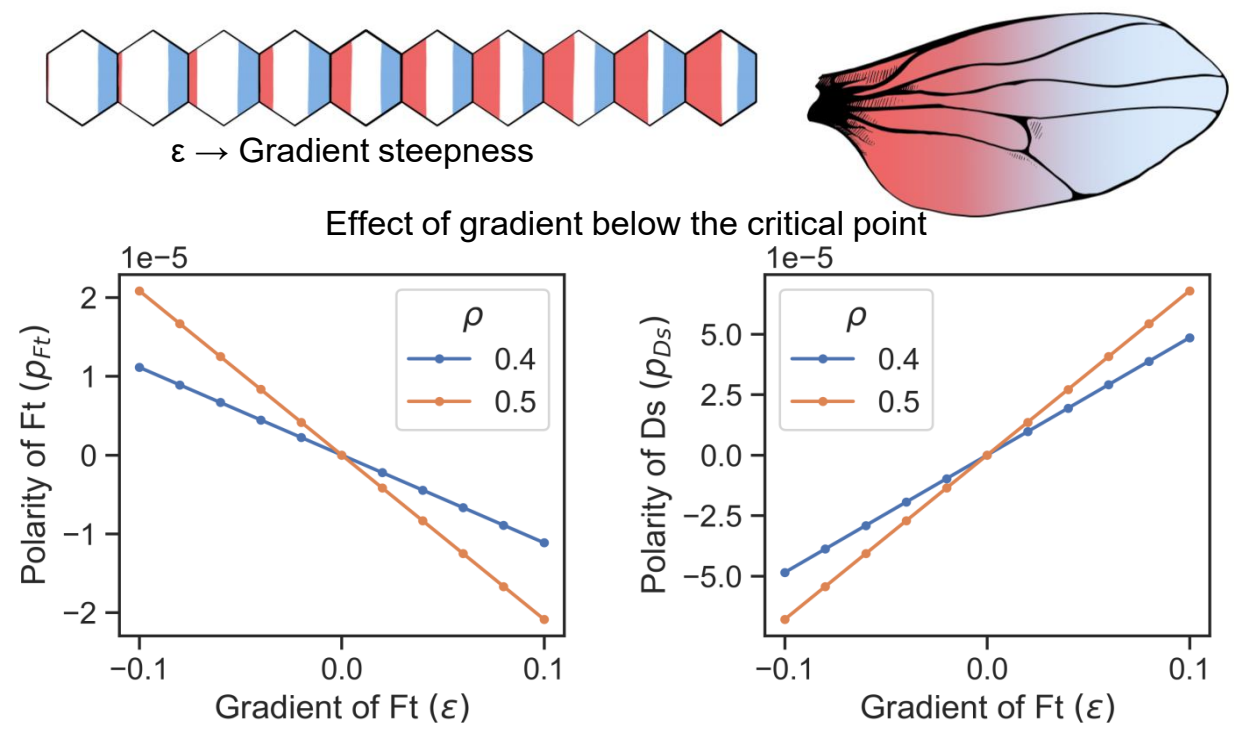

C

Effect of gradient above the critical point
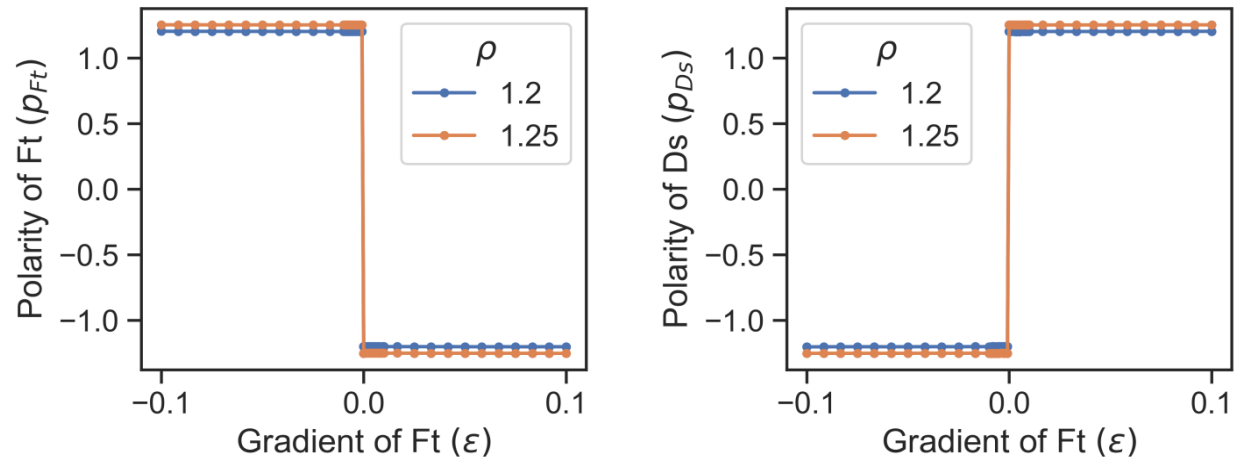

D

Dependence on total protein concentration with different gradients
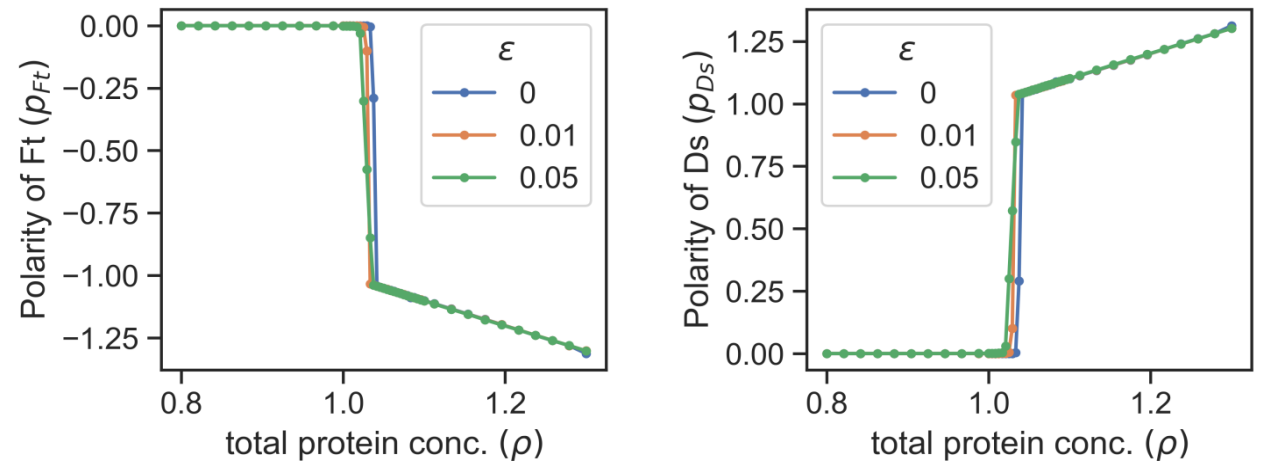

Figure 5. Gradient can polarize the tissue and decide its direction. A) Schematic to describe the model of gradient of expression of Ft (red) in a tissue. B) Polarity of $\mathrm{Ft}\left(p_{F t}\right)$ and $\mathrm{Ds}\left(p_{D s}\right)$ as a function of gradient of $\mathrm{Ft}(\epsilon)$, when total protein concentration $(\rho)$ is below the critical point. The tissue is polarized due to the presence of gradient and the direction is opposite to that of the gradient. C) Polarity of Ft $\left(p_{F t}\right)$ and Ds $\left(p_{D s}\right)$ as a function of gradient $(\epsilon)$ when total protein concentration $(\rho)$ is above the critical point. Here, the gradient decides the direction of the polarity. D) Polarity of Ft $\left(p_{F t}\right)$ and Ds $\left(p_{D s}\right)$ as a function of total protein concentration $(\rho)$ for different values of gradient $(\epsilon)$ 


\section{A}

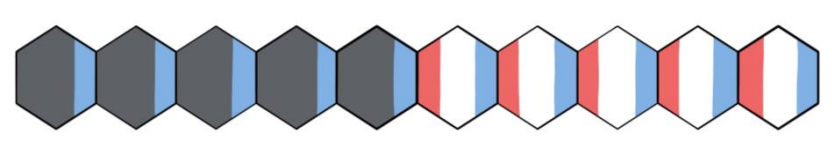

B

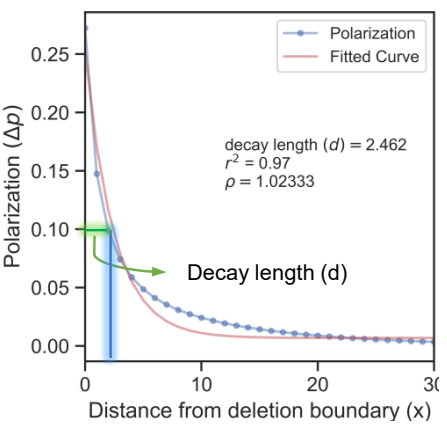

C

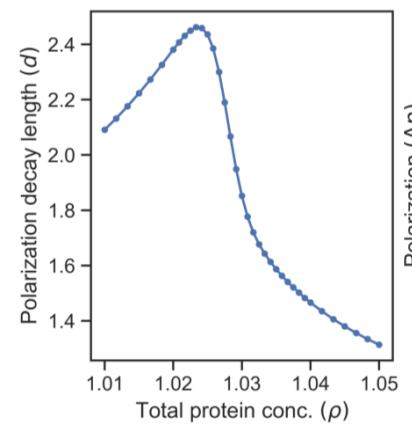

D

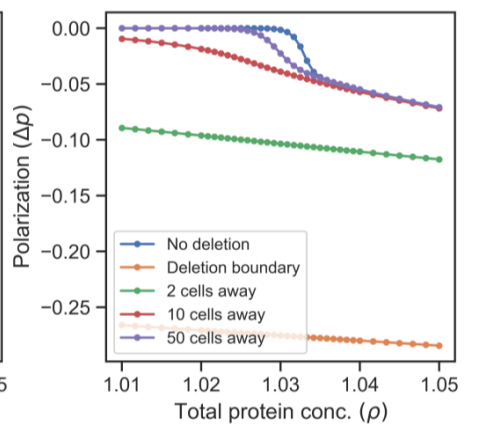

Figure 6. Deletion induces a polarity which decays exponentially. A) Schematic to describe the model of deletion of a gene from one side of the tissue. Ft (red) is deleted from the left side of the tissue. B) Plot of polarity $(\Delta p)$ of each cell as function of distance from the deletion boundary. $(x)$ is measured from the deletion site and corresponds to the number of cells. Polarization decays exponentially. C) Decay length of polarization as a function of total protein concentration. Exponential decay was fit to polarization of 50 cells after the deletion boundary. D) Polarization at different points in the tissue (for different distances from the deletion boundary). No deletion is the control case in which there is no deletion of any gene from the tissue.

and Ds). As expected, as you move away from the deletion boundary (50 cells away), the tissue behaves like the case when there is no deletion at all. (Fig 6 D). To understand why polarity is formed at the deletion boundary and why it follows and exponential decay to we use our continuum model which can be solved analytically. We get:

$$
\Delta p= \begin{cases}0, & \text { if } \rho_{0} \leq 1 \\ \Delta p_{0} e^{-x / d}, & \text { if } \rho_{0}>1\end{cases}
$$

where $d=\left(2 / \rho_{0}\right) \sqrt{(2 \alpha+1)\left(\rho_{0}-1\right)}$, is the decay length of polarity and $\Delta p_{0}$ can be determined by the boundary condition at $x=0$. The continuum model also predicts the exponential decay in polarity which is in agreement with our discrete model results.

Next, we deleted or up-regulated Ft from a circular region of the tissue. The total protein concentration of $\mathrm{Ft}$ is same as that of Ds throughout the tissue but the circular region where it is either zero or a fixed high value (Fig 7 A). We aimed to explain why the experimental results where cells near the deletion boundary are polarized towards or away from the deletion region if a gene is deleted or up regulated in that part respectively $(\mathbf{F i g} 7 \mathrm{~B})$. When $\mathrm{Ft}$ is deleted from a region, and total protein concentration is below the threshold value, the whole tissue gets polarized in arbitrary direction (Fig 7 C). But if the total protein concentration is above the threshold value, the cells polarize in the direction of the deletion $(\mathbf{F i g} 7 \mathrm{D})$. The magnitude of polarization is also greater in this case compared to the case where total protein concentration is below the critical point. When Ft is up-regulated in the region, the results are opposite of the deletion case. Now cells are polarized away from the upregulation region (Fig $7 \mathrm{E}$ and $\mathrm{F}$ ). We can understand these results by simply thinking of the feedback loop. If $\mathrm{Ft}$ is deleted from a region, the $\mathrm{Ft}$ from the surrounding region will make a heterodimer with the Ds of the cells inside the deletion boundary. This will in turn result in localisation of Ds from the surrounding region 
A

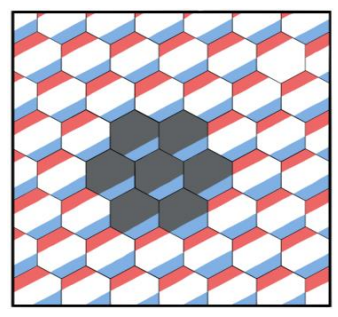

B
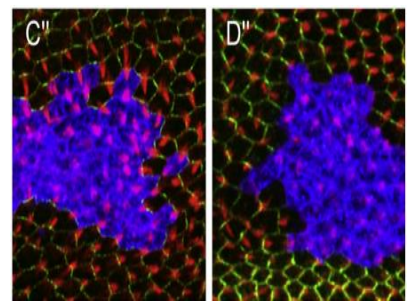

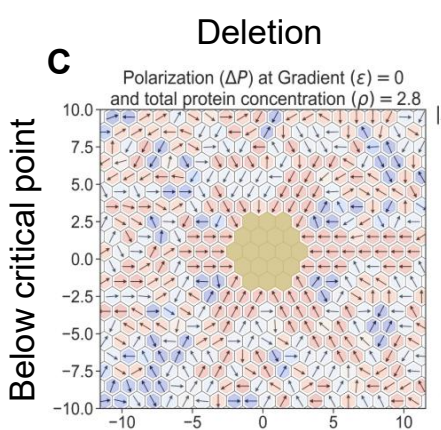

D

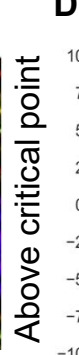

Polarization $(\Delta P)$ at Gradient $(\varepsilon)=0$

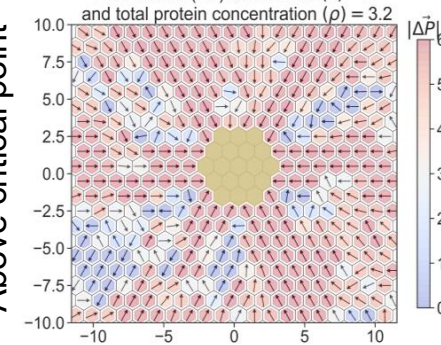

\section{E Upregulation}

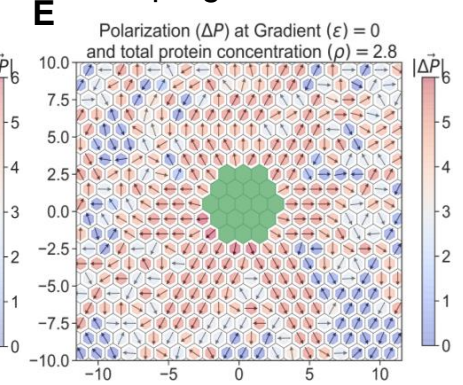

$\mathbf{F}$

Polarization $(\Delta P)$ at Gradient $(\varepsilon)=0$

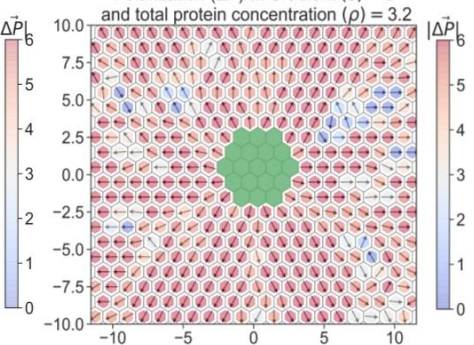

Figure 7. Deletion and upregulation in a 2D layer of tissue. A) Schematic to show deletion of Ft (red) from cells lying in the circular region (grey) while other cells have equal concentration of Ds and Ft. B)Experimental images for deletion/upregulation taken from Chen et al.(2008) 38. Cells around the deletion regions (blue) are polarized in the direction. C) Polarization $(\Delta P)$ when $\mathrm{Ft}$ is removed from a portion of the tissue (yellow) and total protein concentration $(\rho)$ is above the critical point. D) Polarization $(\Delta P)$ when $\mathrm{Ft}$ is removed from a portion of the tissue (yellow) and total protein concentration $(\rho)$ is above the critical point. E) Polarization when Ft is up-regulated in a portion of the tissue (green ) and total protein concentration $(\rho)$ is below the critical point. F)Polarization when Ft is up-regulated in a portion of the tissue (green) and total protein concentration $(\rho)$ is above the critical point.

moving away from deletion region which result in Ft from the next circular layer of cells to localise inwards. This feedback loop will result in polarization of the tissue in the direction of deletion. Similar process happens in the case when Ft is up-regulated except that the directions will be reversed.

\section{Presence of gradient and local down-regulation explains the domineering non-autonomy.}

Finally, we tried understanding one of the most interesting features of PCP. We incorporate both gradient in concentration of $\mathrm{Ft}$ and deleted it from a central region in the tissue (Fig $8 \mathrm{~A})$. We aim to understand the phenomenon of domineering non-autonomy where disruption in polarity due to deletion is seen only on one side of the tissue (Fig 8 B). When we simulated the system, we observe that the cells decide the direction of polarity depending on relative proximity to the deletion region, and strength and direction of gradient. For the cells close to the deletion region, effect of deletion is strong, and they polarize according to the deletion. On one side of the deletion, the gradient supports this polarization and on the other side it opposes the polarization. On the side where it opposes the polarization, cells polarize in direction opposite to the deletion region if they are far enough from the deletion region $(\mathbf{F i g} 8$ C-D). 
bioRxiv preprint doi: https://doi.org/10.1101/2021.11.30.468750; this version posted December 1, 2021. The copyright holder for this preprint (which was not certified by peer review) is the author/funder, who has granted bioRxiv a license to display the preprint in perpetuity. It is made available under aCC-BY-NC-ND 4.0 International license.

A

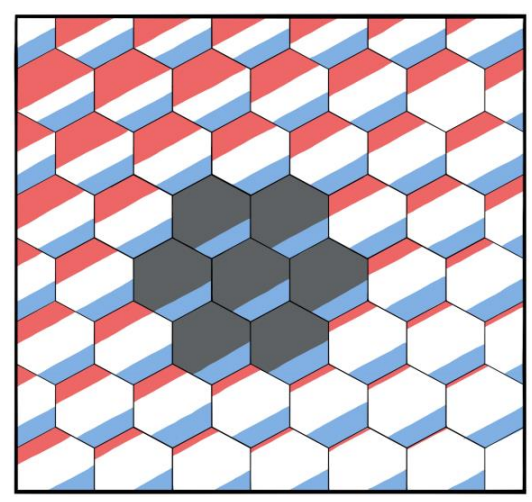

C

Polarization $(\Delta P)$ at Gradient $(\varepsilon)=0.5$

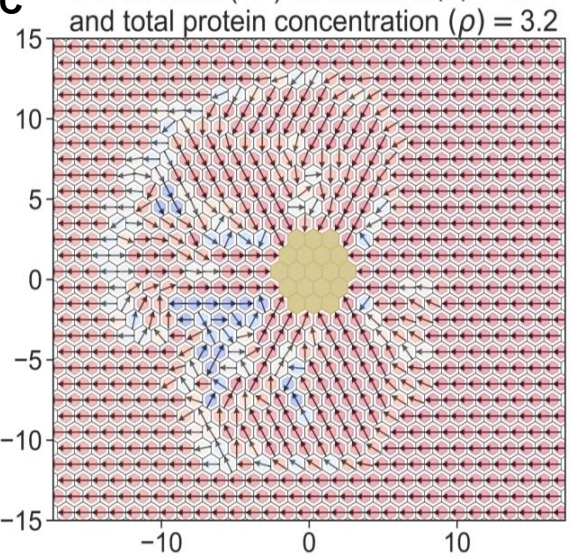

Figure 8. Deletion and gradient can explain domineering non-autonomy. A) Schematic to explain the model of tissue where Ft (red) has been deleted from cells that lie in the circular region (red) and there is a gradient in its expression. B) Experimental image taken from $[20$ to demonstrate domineering non-autonomy $\mathbf{C}$ ) Polarization $(\Delta P)$ in the tissue when gradient $(\epsilon)$ is in the positive direction. The polarity is disrupted only on left side of the deletion region.D) Polarization $(\Delta P)$ in the tissue when gradient $(\epsilon)$ is in the negative direction. The polarity is disrupted only on right side of the deletion region.
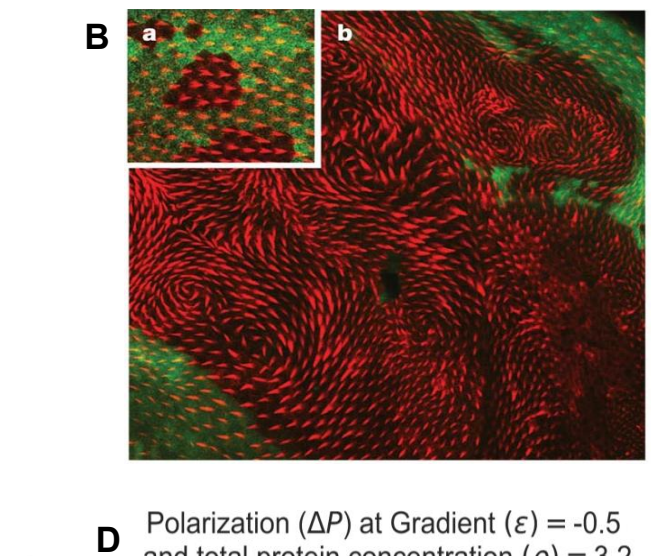

Polarization $(\Delta P)$ at Gradient $(\varepsilon)=-0.5$

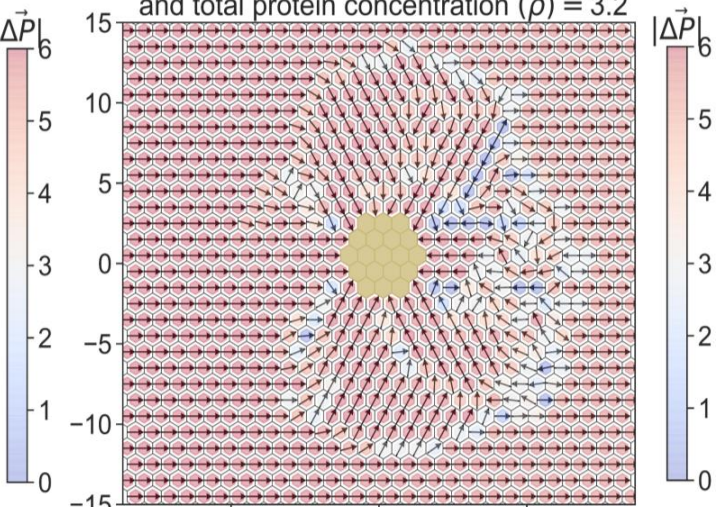
10 and total protein concentration $(\rho)=3.2$ 


\section{Discussion}

The minimal discrete model presented here gives useful framework for combining the role of global (expression gradients) and local (heterodimer formation) interactions in PCP and studying their collective effects. Our framework builds upon the discrete modeling of global/local modules and other phenomenological models.

In this model, we have taken account of simple mechanistic details which are common for both core and global modules. Thus it helps in understanding the properties of both the modules and the predictions from our model can be tested experimentally.

For instance, the exponential decay in polarization near a clone boundary, as predicted by the model, can be measured experimentally. Another experimentally testable prediction from the model is the effect of the protein expression gradients on the non-domineering autonomy where loss of the two proteins, in the presence of expression gradient, can affect the two sides of the clone differently. On the other hand, in absence of a gradient, we observe a disruption in polarity in areas adjacent to the mutant clone but the disruption is isotropic in the sense that the polarization direction near clone boundary is perpendicular to it. This prediction can also be tested experimentally by perturbing gradients and generating mosaic patterns of deletion or overexpression of specific proteins.

Our model also offers advantages over other mechanistic discrete models which have several free parameters making it difficult to analyze [39]. After non-dimensionalizing, we are left with just three free parameters: protein binding rate $(\alpha)$, total protein concentration of Ft and Ds $(\rho)$ and gradient steepness $(\epsilon)$ which are very easy to control and study. In fact, we could also analyze the discrete model in the continuum limit which gives some valuable insights which are validated systematically over a wide range of parameter values in discrete version. Conceptually, the first two parameters ( $\alpha$ and $\rho)$ correspond to the "strength" of relay of PCP "signal" from one cell to another; while the last one $(\epsilon)$ modulates the direction of signal propagation and offers robustness against any stochastic perturbations. In absence of gradient, the polarity is still established in individual cells but is not necessarily coordinated at the tissue level. When the local interaction is weak (total protein concentration is low), the global interaction is responsible for formation and direction of polarity. On the other hand, when local interaction is strong, gradient helps in providing the directional cue and maintenance of polarity in presence of perturbation. Our prediction of gradient providing the robustness against perturbation can be experimentally verified by constructing clones with different gradient strength and measuring polarization in different cases. The clones where strength of gradient is less, we should see more cells which are misaligned compared to the case where the gradient strength is more. This prediction can be extended to claim that polarity establishment in individual cells is not disrupted, but the synchronization is lost. One special case of such disruption is formation of swirling patterns seen in 2D; which the model is able to recapitulate. Thus, the minimal model can explain diverse experimental observations seen in many biological systems; suggesting common design principles at play.

In this model we have only considered the most simplistic and common interactions seen in the proteins involved in PCP. To keep the model simple and widely applicable, we have not taken into account various inter-cellular and intra-cellular interactions which are known in the literature. One of the major limitation of current model is that it doesn't account the change in lattice structure due to cell death, birth and growth. The role of epithelial flow in the PCP has been studied earlier [40]. Future work needs to incorporate these factors in our discrete modelling framework. 


\section{Supporting Information}

\section{Continuum model for one-dimensional model}

This section has been adapted from 34]. Reader is encouraged to refer to the original work for more details and the continuum counterpart of two dimensional model.

In $1 \mathrm{D}$ we can describe the protein distributions by $F t_{L}(x)$ (amount of $F t$ on left edge of cell), $F t_{R}(x), D s_{L}(x)$ and $D s_{R}(x)$. Here $x$ is the cell coordinate. The equations are ( $x$ is not written explicitly)

$$
\begin{aligned}
& \dot{F} t_{L}=\alpha\left(F t_{T}-F t_{0}\right)-\beta F t_{L}+\beta \gamma F t_{L}\left(D s_{R}-l D s_{R}^{\prime}\right) \\
& \dot{F} t_{R}=\alpha\left(F t_{T}-F t_{0}\right)-\beta F t_{R}+\beta \gamma F t_{R}\left(D s_{L}+l D s_{L}^{\prime}\right) \\
& \dot{D} s_{L}=\alpha\left(D s_{T}-D s_{0}\right)-\beta D s_{L}+\beta \gamma D s_{L}\left(F t_{R}-l F t_{R}^{\prime}\right) \\
& \dot{D} s_{R}=\alpha\left(D s_{T}-D s_{0}\right)-\beta D s_{R}+\beta \gamma D s_{R}\left(F t_{L}+l F t_{L}^{\prime}\right)
\end{aligned}
$$

where: $F t_{L}(x), F t_{R}(x), D s_{L}(x), D s_{R}(x)$ represent the amount of protein localized on the left or right edge of the cell. Therefore, they are scalar functions of cell coordinate $x$ in 1D model. $F t_{E}^{\prime}, D s_{E}^{\prime}$ are the derivatives $d F t_{E} / d x$ and $d D s_{E} / d x$ for $E=L, R$ (left and right edges of cell), $F t_{0}(x)=F t_{L}(x)+F t_{R}(x)$ (total membrane bound $F t$ in a cell at location $x$ ) and $D s_{0}=D s_{L}+D s_{R}$, and the quantities $F t_{E}^{\prime}$ and $D s_{E}^{\prime}$ represent the derivatives of $F t_{E}$ and $D s_{E}$ with respect to $x$ where $E=L, R$. The terms $F t_{T}$ and $D s_{T}$ stand for the amount of total (cytoplasmic+membrane bound) proteins in the cell and can be dependent on $x$, the cell location in the tissue.

We can define $p_{F t}=F t_{R}-F t_{L}$ and $p_{D s}=D s_{R}-D s_{L}$ to be the asymmetry $($ or polarity) in the protein localization on cells edges, a measure of PCP. In order to ensure that the protein levels do not become negative at any of the cell edges, this definition of protein asymmetry requires $\left|p_{F t}\right| \leq F t_{0}$ and $\left|p_{D s}\right| \leq D s_{0}$. Using the above equations, we can write the following equations:

$$
\begin{gathered}
\dot{F} t_{0}=2 \alpha\left(F t_{T}-F t_{0}\right)-F t_{0}+\frac{1}{2}\left(F t_{0}\left(D s_{0}-p_{D s}^{\prime}\right)-p_{F t}\left(p_{D s}-D s_{0}^{\prime}\right)\right) \\
\dot{D} s_{0}=2 \alpha\left(D s_{T}-D s_{0}\right)-D s_{0}+\frac{1}{2}\left(D s_{0}\left(F t_{0}-p_{F t}^{\prime}\right)-p_{D s}\left(p_{F t}-F t_{0}^{\prime}\right)\right) \\
\dot{p}_{F t}=-p_{F t}+\frac{1}{2}\left(p_{F t}\left(D s_{0}-p_{F t}^{\prime}\right)+F t_{0}\left(D s_{0}^{\prime}-p_{D s}\right)\right) \\
\dot{p}_{D s}=-p_{D s}+\frac{1}{2}\left(p_{D s}\left(F t_{0}-p_{F t}^{\prime}\right)+D s_{0}\left(F t_{0}^{\prime}-p_{F t}\right)\right)
\end{gathered}
$$

Now, we will deal with the different cases in detail.

\section{Uniform protein expressions}

For the uniform expressions of proteins $F t$ and $D s$ in the tissue, that is $F t_{T}(x)=D s_{T}(x)=\rho$ (a known constant value), we seek homogeneous solutions. For this state, taking a difference of equations (35) and (36) and setting all spatial derivatives to zero gives us $F t_{0}=D s_{0}=\rho_{0}$ (an unknown) for the steady state, and we obtain

$$
\begin{aligned}
\dot{\rho}_{0} & =2 \alpha\left(\rho-\rho_{0}\right)-\rho_{0}+\frac{1}{2}\left(\rho_{0}^{2}-p_{F t} p_{D s}\right) \\
\langle\dot{p}\rangle & =-\langle p\rangle \\
\Delta \dot{p} & =-\left(1-\rho_{0}\right) \Delta p
\end{aligned}
$$


where $\rho_{0}=F t_{0}=D s_{0},\langle p\rangle=p_{F t}+p_{D s}$ and $\Delta p=p_{F t}-p_{D s}$. These equations show that $\langle p\rangle=0$ at the steady state, implying localization of two proteins in opposite directions. In terms of the magnitude of the polarity the tissue can attain two statesunpolarized $(\Delta p=0)$ for $\rho_{0} \leq 1$, and polarized $(\Delta p>0)$ for $\rho_{0}>1$. In the absence of the two constraints, the last equation shows that for $\rho_{0}>1$ the magnitude of the cell polarity increases indefinitely. However, due to $\left|p_{F t}\right| \leq F t_{0}$ and $\left|p_{D s}\right| \leq D s_{0}$ it can be seen that for $\rho_{0}>1$ the two proteins get localized on the two opposite edges of the cells.

\section{Non-uniform protein expressions}

In order to study the effect of gradient, we consider a weak linear 41] gradient of the expression of $F t$ such that $F t_{T}=\rho+\epsilon x$ (with $\epsilon \ll \rho$ ). We assume the following ansatz $F t_{0}=\rho_{0}+F t x, D s_{0}=\rho_{0}+D s x$ with homogeneous polarization level in the tissue. For this case, we get

$$
\Delta \dot{p}=-\left(1-\rho_{0}\right) \Delta p+\frac{\rho_{0}}{2}(D s-F t)
$$

where

$$
F t-D s=\frac{2 \alpha \epsilon}{2 \alpha+1}
$$

For the steady state, we obtain for $\rho_{0} \ll 1$

$$
\Delta p=\frac{\rho_{0}}{2\left(1-\rho_{0}\right)}(D s-F t)
$$

This shows that in case of a tissue-level gradient expression of one of the two proteins, the tissue is always polarized, and that too in a particular direction which depends on the direction of the gradient.

\section{Loss of one protein from a region of the tissue}

In all the analysis till now we considered an infinite 1D tissue. The loss of $F t$ in the region $x<0$ will set the boundary conditions for the unknown variables $F t_{0}, D s_{0}, p_{F t}$ and $p_{D s}$. In this case, we expect $F t_{0}, D s_{0}, p_{F t}$ and $p_{D s}$ to be $x$-dependent and their derivatives with respect to $x$ cannot be ignored. Still, we should get the wildtype behavior as seen above for $x \gg 1$. We presume solutions of type $F t_{0}(x)=\rho_{0}+F t(x)$, $D s_{0}(x)=\rho_{0}+D s(x), p_{F t}(x)=p+p_{F t}(x)$ and $p_{D s}(x)=-p+p_{D s}(x)$. To keep the analysis simpler we also assume $|p| \ll \rho_{0}$. Substituting these values in the equations (35) and (36) and solving the equations for the steady state, we obtain

$$
\Delta p^{\prime \prime}=-(2 \alpha+1)\left(1-\rho_{0}\right)\left(\frac{2}{\rho_{0}}\right)^{2} \Delta p
$$

In order to ensure $\Delta p \rightarrow 0$ as $x \rightarrow \infty$, we need $\rho_{0}>1$ which gives

$$
\Delta p= \begin{cases}0, & \text { if } \rho_{0} \leq 1 \\ \Delta p_{0} e^{-x / d}, & \text { if } \rho_{0}>1\end{cases}
$$

where $d=\left(2 / \rho_{0}\right) \sqrt{(2 \alpha+1)\left(\rho_{0}-1\right)}$, and $\Delta p_{0}$ can be determined by the boundary condition at $x=0$. This demonstrates an exponential decay in the magnitude of cell polarization from the boundary of the region where one of the two proteins is not expressed. 


\section{Supplementary Figures}
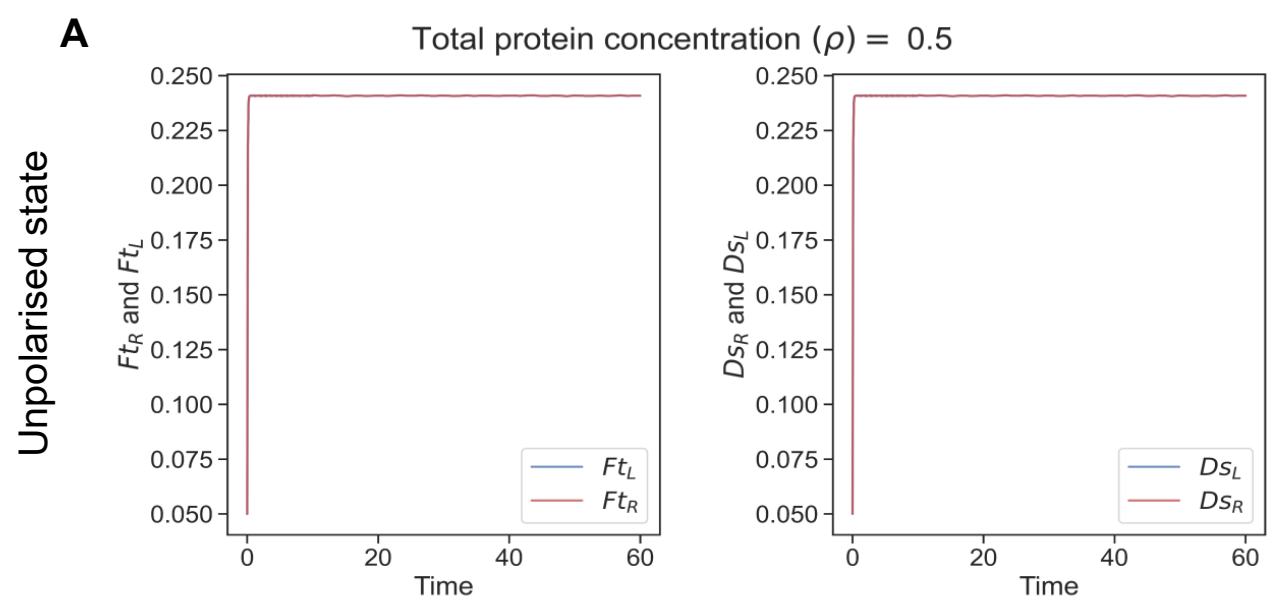

B

Total protein concentration $(\rho)=1.5$
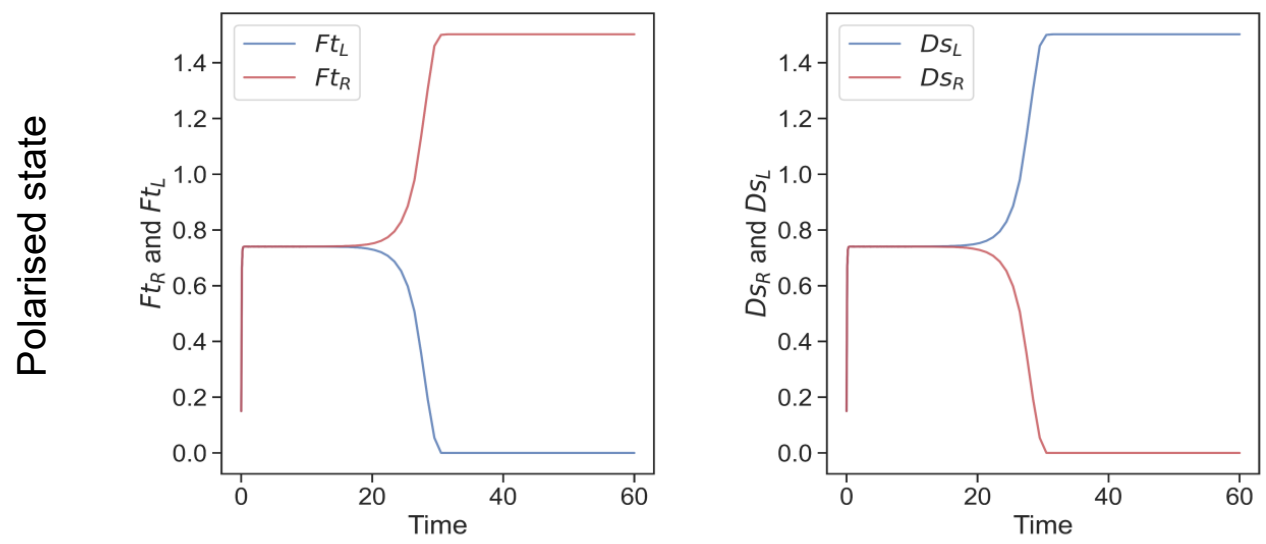

Figure S1. Dynamics in 1D: Level of Ft and Ds in left and right side $\left(F t_{L}, F t_{R}, D s_{L}\right.$ and $D s_{R}$ ) of the tissue as a function of time. A) When the total protein concentration of Ft and Ds $(\rho)$ is below the critical value both proteins localise equally on both sides of the cell. B) But when the total protein concentration of Ft and Ds $(\rho)$ is above the critical value, we observe that the proteins localise completely to one side of the tissue. 

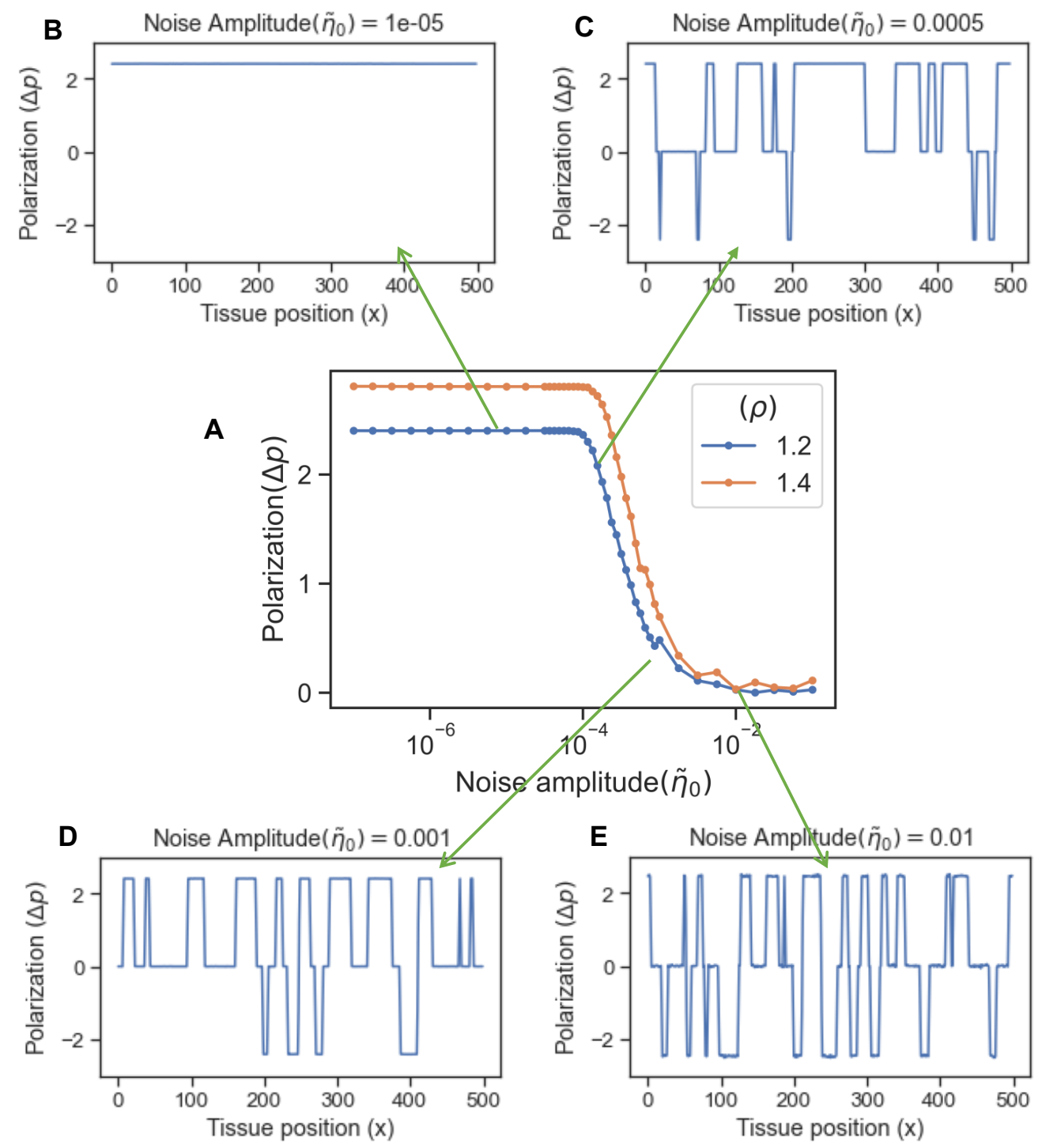

Figure S2. Effect of noise in protein kinetics on polarization A) Polarization $(\Delta p)$ as function of noise amplitude $\left(\tilde{\eta}_{0}\right)$ for different total protein concentrations $(\rho)$. B) Polarization $(\Delta p)$ in the tissue when the level of noise is negligible $\left(10^{-5}\right)$. C) Polarization at high amplitude of noise $\left(\tilde{\eta}_{0}=5 * 10^{-4}\right)$, some parts of the tissue are aligned in the opposite direction $\mathbf{D})$ At higher noise amplitude $\left(\tilde{\eta}_{0}=10^{-3}\right)$, the average polarity of the tissue reduces $\mathbf{E})$ At very high noise amplitude $\left(\tilde{\eta}_{0}=10^{-} 2\right)$, the tissue loses overall polarization as different parts of the tissue are aligned in opposite direction. 

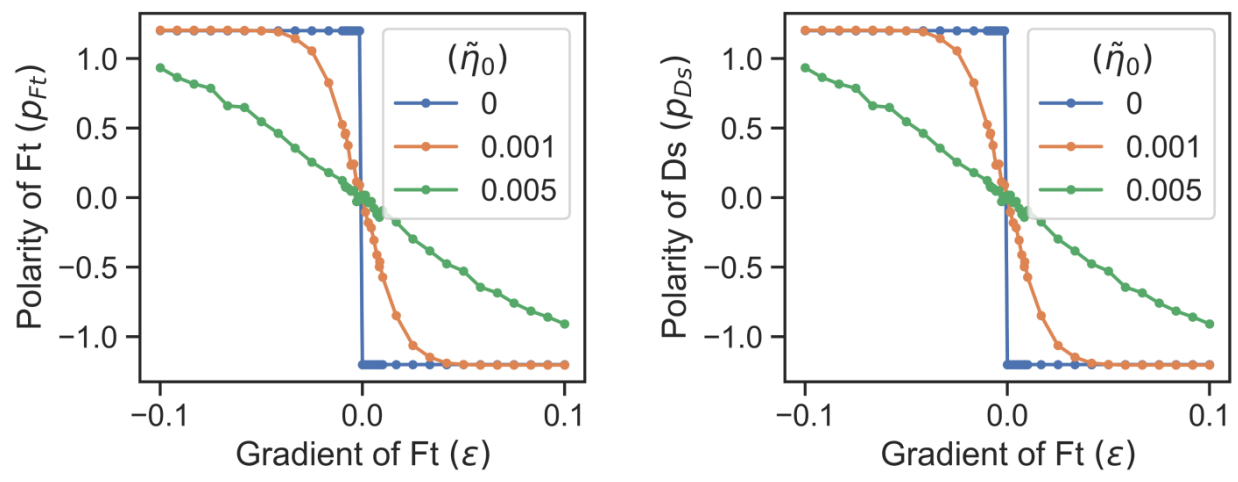

B Effect of when noise in presence and absence of gradient
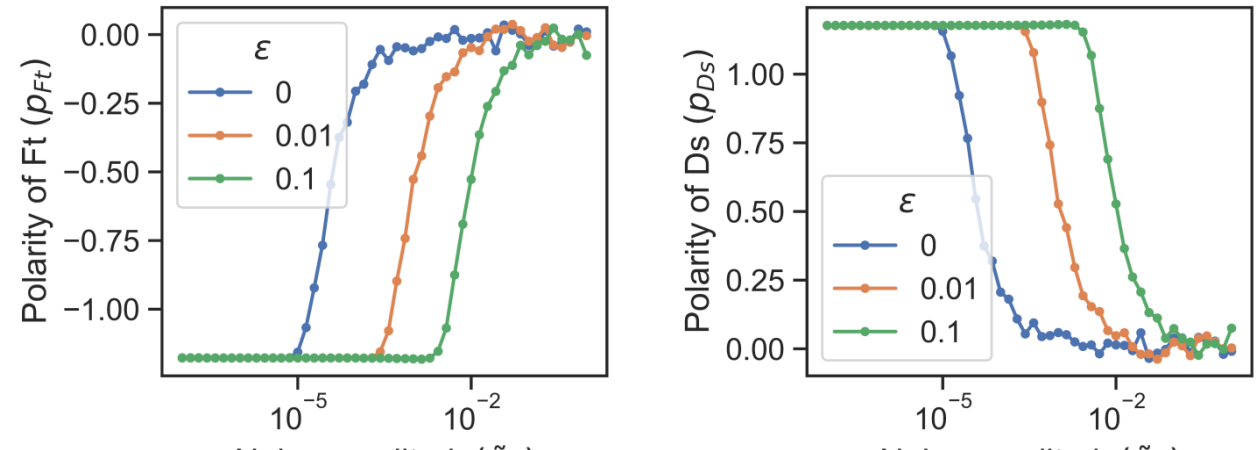

Figure S3. Effect of noise in protein kinetics on the polarization in presence of gradient cue. A) Polarization of $\mathrm{Ft}(p f t)$ and $\mathrm{Ds}(p d s)$ as a function of gradient $(\epsilon)$ in presence of noise $\left(\tilde{\eta}_{0}=0.001,0.005\right)$ and without it $\left(\tilde{\eta}_{0}=0\right)$. B) Polarization of $\mathrm{Ft}\left(p_{f t}\right)$ and Ds $(p d s)$ as a function of noise amplitude $\left(\tilde{\eta}_{0}\right)$ in presence of gradient $(\epsilon=0.01,0.1)$ and without it $(\epsilon=0)$. 

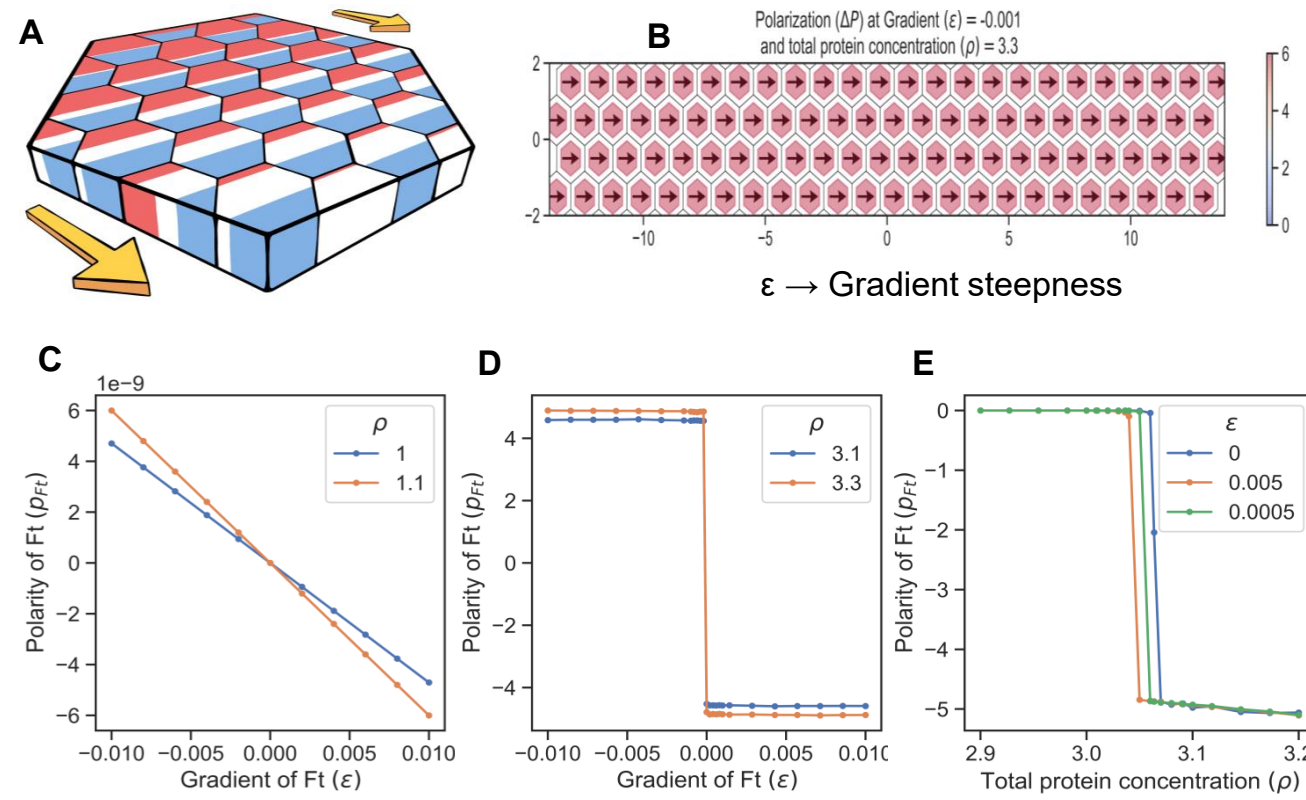

D
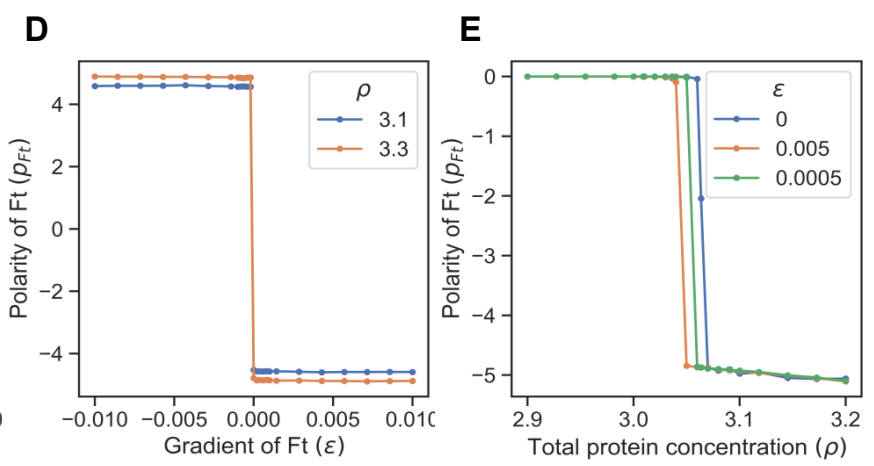

Figure S4. Gradient decides the direction and maintains polarization in 2D: A) Schematic of model of a tissue where Ft (red) is expressed in a gradient and Ds (blue) is expressed uniformly throughout the tissue. B) Polarization $(\Delta P)$ in the tissue when gradient is present. C) Polarization of Ft $\left(p_{\mathrm{Ft}}\right)$ as a function of gradient of $\mathrm{Ft}$ $(\epsilon)$ when the total protein concentration of Ft and Ds $(\rho)$ is below the critical point. D) Polarization of Ft $\left(p_{\mathrm{Ft}}\right)$ as a function of gradient of $\mathrm{Ft}(\epsilon)$ when the total protein concentration of Ft and Ds $(\rho)$ is above the critical point. E) Polarization of Ft $\left(p_{\mathrm{Ft}}\right)$ as a function of total protein concentration of $\mathrm{Ft}$ and Ds $(\rho)$ for different value of gradient of $\mathrm{Ft}(\epsilon)$.

\section{S2 Videos}

\section{Non-homogeneous polarization:}

Non-uniform polarization in 2D without gradient. Swirling patterns and vortices are formed. Periodic boundary conditions and initial conditions are such that there is small misalignment.

\section{Polarization in case of deletions:}

One of the genes is deleted from the central part of the tissue. The system gets polarised in the direction of the deletion region.

\section{2-D Polarization in case of deletion with gradient:}

One of the proteins is deleted from the central region of the tissue. There is also a gradient across the tissue

\section{Acknowledgments}

We would like to acknowledge Atchuta Srinivas Duddu for the schematics. We would also like to acknowledge and thank Prof Sriram Ramaswamy for his constant valuable support and inputs. D.S. is supported by KVPY fellowship awarded by Department of Science and Technology (DST), Government of India. MKJ is supported by Ramanujan Fellowship (SB/S2/RJN 049/2018) awarded by the Science and Engineering Research Board (SERB), DST, Government of India. MSR would like to acknowledge the financial support provided by the seed grant from IIT Hyderabad. 


\section{References}

1. Núria Torras, María García-Díaz, Vanesa Fernández-Majada, and Elena Martínez. Mimicking epithelial tissues in three-dimensional cell culture models. Frontiers in Bioengineering and Biotechnology, 0:197, 122018.

2. M. T. Butler and J. B. Wallingford. Planar cell polarity in development and disease. Nat. Rev. Mol. Cell Biol., 18(6):375-388, 062017.

3. Eugenia Papakrivopoulou, Daniyal J. Jafree, Charlotte H. Dean, and David A. Long. The biological significance and implications of planar cell polarity for nephrology. Frontiers in Physiology, 0:180, 2021.

4. Helen Strutt and David Strutt. How do the fat-dachsous and core planar polarity pathways act together and independently to coordinate polarized cell behaviours? Open Biology, 11, 22021.

5. E. K. Vladar, R. D. Bayly, A. M. Sangoram, M. P. Scott, and J. D. Axelrod. Microtubules enable the planar cell polarity of airway cilia. Curr Biol, 22(23):2203-2212, Dec 2012.

6. Michael R. Deans. Conserved and divergent principles of planar polarity revealed by hair cell development and function. Frontiers in Neuroscience, 0:1387, 102021.

7. Xiaofei Li, Daogong Zhang, Lei Xu, Yuechen Han, Wenwen Liu, Wei Li, Zhaomin Fan, Richard M. Costanzo, III Jerome F. Strauss, Zhibing Zhang, and Haibo Wang. Planar cell polarity defects and hearing loss in sperm-associated antigen 6 (spag6)-deficient mice. https://doi.org/10.1152/ajpcell.00166.2020, 320:C132-C141, 12021.

8. Y. Mao, A. L. Tournier, P. A. Bates, J. E. Gale, N. Tapon, and B. J. Thompson. Planar polarization of the atypical myosin Dachs orients cell divisions in Drosophila. Genes Dev, 25(2):131-136, Jan 2011.

9. Amy L. Brittle, Ada Repiso, José Casal, Peter A. Lawrence, and David Strutt. Four-jointed modulates growth and planar polarity by reducing the affinity of dachsous for fat. Current Biology, 20:803-810, 52010.

10. Hiroyuki O. Ishikawa, Hideyuki Takeuchi, Robert S. Haltiwanger, and Kenneth D. Irvine. Four-jointed is a golgi kinase that phosphorylates a subset of cadherin domains. Science, 321:401-404, 72008.

11. Rosalind Hale, Amy L. Brittle, Katherine H. Fisher, Nicholas A.M. Monk, and David Strutt. Cellular interpretation of the long-range gradient of four-jointed activity in the drosophila wing. eLife, 2015, 22015.

12. Maja Matis and J. D. Axelrod. Regulation of pcp by the fat signaling pathway. Genes Dev, 27(20):2207-2220, 102013.

13. P. A. Lawrence and J. Casal. Planar cell polarity: two genetic systems use one mechanism to read gradients. Development, 145(23), Dec 2018.

14. Wen Yih Aw and Danelle Devenport. Planar cell polarity: global inputs establishing cellular asymmetry. Current Opinion in Cell Biology, 44:110-116, 2 2017. 
15. Bo Gao, Hai Song, Kevin Bishop, Gene Elliot, Lisa Garrett, Milton A. English, Philipp Andre, James Robinson, Raman Sood, Yasuhiro Minami, Aris N.

Economides, and Yingzi Yang. Wnt signaling gradients establish planar cell polarity by inducing vangl2 phosphorylation through ror2. Developmental Cell, 20:163-176, 22011.

16. Y. Yang and M. Mlodzik. Wnt-Frizzled/planar cell polarity signaling: cellular orientation by facing the wind (Wnt). Annu Rev Cell Dev Biol, 31:623-646, 2015.

17. D. Devenport. The cell biology of planar cell polarity. J Cell Biol, 207(2):171-179, Oct 2014.

18. M. Simons and M. Mlodzik. Planar cell polarity signaling: from fly development to human disease. Annu Rev Genet, 42:517-540, 2008.

19. Saw Myat Thanda W. Maung and Andreas Jenny. Planar cell polarity in drosophila. http://dx.doi.org/10.4161/org.7.3.18143, 7:165-179, 2011.

20. D. Ma, C. H. Yang, H. McNeill, M. A. Simon, and J. D. Axelrod. Fidelity in planar cell polarity signalling. Nature, 421(6922):543-547, Jan 2003.

21. Peter A. Lawrence, Gary Struhl, and José Casal. Planar cell polarity: one or two pathways? Nature Reviews Genetics 2007 8:7, 8:555-563, 62007.

22. Katherine H. Fisher, David Strutt, and Alexander G. Fletcher. Experimental and theoretical evidence for bidirectional signaling via core planar polarity protein complexes in drosophila. iScience, 17:49-66, 72019.

23. Wen Yih Aw and Danelle Devenport. Planar cell polarity: global inputs establishing cellular asymmetry. Current opinion in cell biology, 44:110-116, 2017.

24. K. Amonlirdviman, N. A. Khare, D. R. P. Tree, W.-S. Chen, J. D. Axelrod, and C. J. Tomlin. Mathematical modeling of planar cell polarity to understand domineering nonautonomy . Science, 307(5708):423-426, 2005.

25. Mohit Kumar Jolly, Mohd Suhail Rizvi, Amit Kumar, and Pradip Sinha. Mathematical modeling of sub-cellular asymmetry of fat-dachsous heterodimer for generation of planar cell polarity. PLOS ONE, 9(5):1-10, 052014.

26. M. Mani, S. Goyal, K. D. Irvine, and B. I. Shraiman. Collective polarization model for gradient sensing via Dachsous-Fat intercellular signaling. Proc. Natl. Acad. Sci. U.S.A., 110(51):20420-20425, Dec 2013.

27. Y. Burak and B. I. Shraiman. Order and stochastic dynamics in Drosophila planar cell polarity. PLoS Comput Biol, 5(12):e1000628, Dec 2009.

28. L. D. Hazelwood and J. M. Hancock. Functional modelling of planar cell polarity: an approach for identifying molecular function. BMC Dev. Biol., 13:20, May 2013.

29. Shahriar Shadkhoo and Madhav Mani. The role of intracellular interactions in the collective polarization of tissues and its interplay with cellular geometry. PLOS Computational Biology, 15(11):1-29, 112019.

30. J. D. Axelrod and C. J. Tomlin. Modeling the control of planar cell polarity. Wiley Interdiscip Rev Syst Biol Med, 3(5):588-605, 2011.

31. J. Casal, P. A. Lawrence, and G. Struhl. Two separate molecular systems, Dachsous/Fat and Starry night/Frizzled, act independently to confer planar cell polarity. Development, 133(22):4561-4572, Nov 2006. 
32. Amy Brittle, Chloe Thomas, and David Strutt. Planar polarity specification through asymmetric subcellular localization of fat and dachsous. Current Biology, 22:907-914, 52012 .

33. Abhijit A. Ambegaonkar, Guohui Pan, Madhav Mani, Yongqiang Feng, and Kenneth D. Irvine. Propagation of dachsous-fat planar cell polarity. Current Biology, 22:1302-1308, 72012.

34. Mohd Suhail Rizvi, Divyoj Singh, Mohit Kumar Jolly, and Sriram Ramaswamy. Continuum Theory for Planar Cell Polarity. Submitted, 2021.

35. Gábor Balázsi, Alexander Van Oudenaarden, and James J. Collins. Cellular decision making and biological noise: From microbes to mammals. Cell, 144:910-925, 32011.

36. Jeffrey D. Axelrod. Planar cell polarity signaling in the development of left-right asymmetry. Current Opinion in Cell Biology, 62:61-69, 22020.

37. H. Matakatsu and S. S. Blair. Interactions between Fat and Dachsous and the regulation of planar cell polarity in the Drosophila wing. Development, 131(15):3785-3794, Aug 2004.

38. Wei-Shen Chen, Dragana Antic, Maja Matis, Catriona Y. Logan, Michael Povelones, Graham A. Anderson, Roel Nusse, and Jeffrey D. Axelrod. Asymmetric Homotypic Interactions of the Atypical Cadherin Flamingo Mediate Intercellular Polarity Signaling. Cell, 133(6):1093-1105, jun 2008.

39. Ryan N. Gutenkunst, Joshua J. Waterfall, Fergal P. Casey, Kevin S. Brown, Christopher R. Myers, and James P. Sethna. Universally sloppy parameter sensitivities in systems biology models. PLOS Computational Biology, 3:e189, 10 2007.

40. Benoît Aigouy, Reza Farhadifar, Douglas B. Staple, Andreas Sagner, Jens-Christian Röper, Frank Jülicher, and Suzanne Eaton. Cell flow reorients the axis of planar polarity in the wing epithelium of drosophila. Cell, 142(5):773-786, 2010.

41. P. A. Lawrence, G. Struhl, and J. Casal. Do the protocadherins Fat and Dachsous link up to determine both planar cell polarity and the dimensions of organs? Nat Cell Biol, 10(12):1379-1382, Dec 2008. 